Pacific Journal of Mathematics

A NEW KIND OF EIGENFUNCTION EXPANSIONS ON GROUPS 


\section{A NEW KIND OF EIGENFUNCTION EXPANSIONS ON GROUPS}

\section{HORST LEPTIN}

Let $G$ be a locally compact group, $C_{\infty}(G)$ the Banach algebra of $C$-valued continuous functions on $G$ vanishing at infinity, and let $\mathscr{2}$ be a translation-invariant dense $*$-subalgebra. We assume that $\mathscr{Q}$ has its own norm, such that it is a Banach $G$-algebra with involution. Then the twisted convolution algebra $\mathscr{L}=L^{1}(G, \mathscr{2})$ is simple and symmetric and there exists - up to unitary equivalence - exactly one irreducible $*$-representation $\lambda$, mapping $\mathscr{L}$ into the compact operators of $L^{2}(G)$. Thus for hermitian $f \in \mathscr{L}$ one has the canonical spectral decomposition $\lambda(f)=$ $\sum_{j} \alpha_{j} E_{j}$ with $\left\{\alpha_{j}\right\}=\operatorname{Spec} \lambda(f)=\operatorname{Spec}_{\mathscr{L}}(f), E_{j}$ finite-dimensional projections in $L^{2}(G)$. It turns out that $E_{j}=\lambda\left(e_{j}\right)$ for idempotent $e_{j} \in \mathscr{L}$, hence every hermitian $f \in \mathscr{L}$ defines uniquely a Fourier series $\sum \alpha_{j} e_{J}$ in $\mathscr{L}$. Different convergence properties of such expansions are studied.

The main result states that for "radial functions" $f$ the eigenfunctions $e_{J}$ span a maximal commutative subalgebras of $\mathscr{L}$ and that there exists a summation method for these $f$, generalizing the Fejer kernel for periodic functions. More precisely: There exists a bounded approximate identity for $\mathscr{L}$, consisting of finite linear combinations of the $e_{j}$. Applications are given to algebras $L^{1}(N)$ for nilpotent Lie groups $N$, in particular all such $N$ are determined, on which a compact abelian group $K$ acts such that the subalgebra $L_{K}^{1}(N)$ of radial (i.e. $K$-invariant) functions is commutative.

Let $G$ be a locally compact group with a liminal $C^{*}$-group-algebra. Then every irreducible unitary representation $\pi$ of $G$, resp. of $L^{1}(G)$, maps $L^{2}(G)$ into the compact operators of the Hilbert space $\mathscr{H}(\pi)$. Thus for a hermitian function $f \in L^{1}(G)$ the operator $\pi(f)$ has a spectral decomposition $\pi(f)=\sum_{0}^{\infty} \alpha_{j} E_{j}$ with orthogonal minimal projectors $E_{j}$ of $\mathscr{H}(\pi)$. It can happen that also the $E_{i}$ are in the image $\pi\left(L^{1}\right)$, i.e. $E_{i}=\pi\left(e_{i}\right)$ with $e_{i} \in L^{1}(G)$. In this case it is reasonable to say that

$$
f \sim \sum_{i=0}^{\infty} \alpha_{i} e_{i} \quad(\bmod \operatorname{ker} \pi)
$$

is an eigenfunction expansion modulo $\pi$ of $f$, and to ask in which sense the series $\sum \alpha_{j} e_{j}$ converges to $f$.

To give an example let us take for $G$ the Mackey group of $H=\mathbf{T} \times \mathbf{Z}$, $\mathbf{T}$ the circle group, with respect to the cocycle $c((\zeta, m),(\vartheta, n))=\vartheta^{m}$. Thus $G=H \times \mathbf{T}$ with product $(x, \alpha)(y, \beta)=(x y, c(x, y) \alpha \beta)$. The group $G$ is 
nilpotent of class 2 and has essentially one infinite dimensional irreducible representation $\pi$ with $\mathscr{H}(\pi)=L^{2}(\mathbf{T})$, defined by

$$
(\pi(\zeta, n, \lambda) \phi)(\zeta)=\bar{\lambda}(\bar{\zeta} \xi)^{n} \phi(\bar{\zeta} \xi) .
$$

It is easy to see that the quotient $L^{1}(G) / \operatorname{ker} \pi$ is isomorphic to $L^{1}(H, c)$, i.e. the $L^{1}$-algebra of $H$, but with the twisted convolution defined with the aid of the cocycle $c$. This algebra in turn is isomorphic with the twisted product algebra $L^{1}(\mathbf{T}, A(\mathbf{T}))$, where $A(\mathbf{T})$ is the Fourier algebra of $\mathbf{T}$, i.e. the algebra of all functions on $\mathbf{T}$ with absolutely convergent Fourier series. The product is defined by

$$
(f * g)(\zeta, \vartheta)=\int_{\mathbf{T}} f(\zeta \xi, \bar{\xi} \vartheta) g(\bar{\xi}, \vartheta) d \xi
$$

for details see e.g. [7] or [9]. Then $\pi$ maps $L^{1}(\mathbf{T}, A(\mathbf{T}))$ faithfully into the compact operators of $L^{2}(\mathbf{T})$. Now $\mathbf{C} \subset A(\mathbf{T})$, hence $L^{1}(\mathbf{T}) \subset L^{1}(\mathbf{T}, A(\mathbf{T}))$ and actually the ordinary $L^{1}(\mathbf{T})$ is in this natural way a subalgebra of $L^{1}(\mathbf{T}, A(\mathbf{T}))$. It is not hard to see that for $f \in L^{1}(\mathbf{T})$ the series (1) is exactly the classical Fourier series of the function $f$. This example shows that general nontrivial results about convergence questions of series (1) may be difficult to obtain. In this paper we will mainly treat the case of the algebras $\mathscr{L}=L^{1}(G, \mathscr{Q})$ for $\mathscr{Q} \subset C_{\infty}(G)$. These $\mathscr{L}$ 's have been introduced in [7] and extensively studied in [9], in particular [9] contains a complete description of the ideal theory of $\mathscr{L}$. Probably the most important cases of these $\mathscr{L}$ 's are the algebras $\Gamma\left(\mathbf{R}^{n}\right)=L^{1}\left(\mathbf{R}^{n}, A\left(\mathbf{R}^{n}\right)\right)$, which are exactly the primitive quotients of the group algebras $L^{1}(G)$ for nilpotent connected Lie groups of class 2 . In these cases there is a natural notion of radial functions and it is this class of functions for which we can prove satisfactory results. As an application of the results we will explicitly determine all connected nilpotent groups $G$ with a continuous action of a torus $K=\mathbf{T}^{n}$, for which the invariant functions form a commutative subalgebra $L_{K}^{1}(G)$. For $G=\mathbf{H}^{m}$, the $2 m+1$ dimensional Heisenberg group, these $L_{K}^{1}\left(\mathbf{H}^{n}\right), K=\mathbf{T}^{n}$, have recently attracted some interest, see e.g. [1], [4], [5], [13]. In [13] also more general nilpotent groups are studied. It is not hard to see that at least some of the results in [13] are easy consequences of this paper.

We are interested in the twisted algebras

$$
\mathscr{L}=L^{1}(G, \mathscr{Q}) \text {. }
$$

Here $G$ is a locally compact group with left Haar measure $d x$ and $\mathscr{Q}$ a subalgebra of $C_{\infty}(G)$, the algebra of all $\mathbf{C}$-valued continuous functions on $G$, vanishing at infinity, with the usual norm $|f|_{\infty}$. The algebra $\mathscr{Q}$ is always 
assumed to be closed under complex conjugation $f \rightarrow f^{*}, f^{*}(x)=\overline{f(x)}$, and left translation and to have its own norm $|f|$ with $|f| \geq|f|_{\infty}$, so that 2 is a Banach algebra with respect to $|f|$. Moreover, as in [8], §2, or [11], Theorem 4, we assume that $\left|f^{*}\right|=|f|,\left|f^{z}\right|=\left|f_{z}\right|=|f|$ (for $f^{z}(x)=f(z x)$, $\left.f_{z}(x)=f(x z)\right), z \rightarrow f^{z}$ is continuous from $G$ into $\mathscr{Q}$, the compactly supported functions $\mathscr{Q}_{0}$ are dense in $\mathscr{2}$ and finally, the $C^{*}$-hull $\mathscr{2}^{*}$ of $\mathscr{Q}$ coincides naturally with $C_{\infty}(G)$. Under these conditions $\mathscr{L}$ is a simple and symmetric involutive Banach algebra with essentially only one irreducible unitary representation $\lambda$, realized in $L^{2}(G)$ by

$$
\lambda(f) \xi(x)=f * \xi(x)=\int f\left(x y, y^{-1}\right) \xi\left(y^{-1}\right) d y .
$$

Here we consider $f \in \mathscr{L}$ as a function on $G \times G: f(x, y)=f(x)(y)$. The same formula defines irreducible representations $\lambda^{p}$ in $L^{p}(G)$ for all $p$ with $1 \leq p<\infty$.

The finite rank elements form a two-sided dense ideal $\mathscr{E}$. The elements of rank 1 have been explicitly determined in [9], Theorems 1 and 2. They are of the form $f=a \circ b$ with some $a, b \in \mathscr{Q}$, for which $a \circ b$ is defined by (see [9], p. 128)

$$
(a \circ b)(x, y)=\Delta(y) a(x y) \overline{b(y)}
$$

The subset $\mathscr{Q}_{1}$ of all $a \in \mathscr{Q}$ with $a \circ a \in \mathscr{L}$ is a Segal algebra in $\mathscr{Q}$ with norm $\|a\|=|a \circ u|_{1}$ for a fixed $u \in \mathscr{Q}_{1}$ with $|u|_{2}=1$, on which $G$ acts continuously and isometrically. Moreover, for every real $p, 1 \leq p<\infty, \mathscr{Q}_{1}$ is contained in $L^{p}(G)$ and the injection $\mathscr{Q} \rightarrow L^{p}$ is bounded. One of the results in [9] states that the closed left ideals in $\mathscr{L}$ are in a canonic $1: 1$ correspondence with the closed subspaces of $\mathscr{Q}_{1}$.

Now let $f$ be a hermitian element in $\mathscr{L}$. Then $\lambda(f)$ is a selfadjoined compact operator, hence $\lambda(f)=\sum \alpha_{j} E_{j}$ with pairwise orthogonal hermitian projectors $E_{j}$ of finite rank and $\alpha=\left\{\alpha_{j}\right\}$ the spectrum of $\lambda(f)$. As $\mathscr{L}$ is symmetric $\alpha$ is also the spectrum of $f$ in $\mathscr{L}$, hence if $R(\zeta)$ is the resolvent of $f$ in $\mathscr{L}$, and $\mathrm{c}$ a circle with center $\alpha_{j}$ and so that no other $\alpha_{k} \in \alpha$ is inside $\mathrm{c}$, then

$$
\frac{1}{2 \pi i} \int_{c} R(\zeta) d \zeta=f_{J}
$$

defines an element $f_{j}$ in $\mathscr{L}$ with

$$
\lambda\left(f_{j}\right)=\frac{1}{2 \pi i} \int_{c} \lambda(R(\zeta)) d \zeta=E_{j},
$$

because $\lambda(R(\zeta))$ is the resolvent of $\lambda(f)$. It follows that $f_{j} \in \mathscr{E}$, thus

$$
f_{j}=\sum_{k=1}^{m_{j}} v_{j k} \circ v_{j k}
$$


with $v_{j k} \in \mathscr{Q}_{1},\left\{v_{j k}{ }^{\circ} v_{j k}\right\}_{j, k}$ a system of pairwise orthogonal hermitian idempotents of rank 1 in $\mathscr{L}$. We have proved (see [10], "Satz" on p. 138):

Proposition 1. Let $f=f^{*}$ be a hermitian element in $\mathscr{L}$. The spectrum of $f$ in $\mathscr{L}$ is a real sequence $\left\{\alpha_{j}\right\}$, finite or converging to 0 . There exists a corresponding sequence $\left\{f_{j}\right\}$ of pairwise orthogonal hermitian idempotents in $\mathscr{E}$ with

$$
\lambda(f)=\sum \alpha_{j} \lambda\left(f_{j}\right)
$$

in the compact operators $\mathscr{K}\left(L^{2}(G)\right)$.

Obviously the sequences $\left\{\boldsymbol{\alpha}_{j}\right\}$ and $\left\{f_{j}\right\}$ are uniquely defined by $f$, therefore we define

Definition. Let $f$ be a normal element in $\mathscr{L}$ (i.e. $f * f^{*}=f^{*} * f$ ) with spectrum $\left\{\alpha_{j}\right\}$. Let the sequence $\left\{f_{j}\right\}$ of finite rank idempotents in $\mathscr{L}$ be defined by $f$ as described in Proposition 1 (i.e. $\lambda(f)=\sum \alpha_{j} \lambda\left(f_{j}\right), \alpha_{j} \in \mathbf{C}$ ). We write

$$
f \sim \sum \alpha_{j} f_{j}
$$

and call $\sum \boldsymbol{\alpha}_{j} f_{j}$ the Fourier series of $f$.

The example discussed in the introduction shows that this definition indeed generalizes the classical notion of the Fourier series of a periodic function.

We have norm convergence of the image $\sum \boldsymbol{\alpha}_{i} \lambda\left(f_{i}\right)$ of the Fourier series to $\lambda(f)$, but quite evidently we cannot expect the same for $f$ and its series in $\mathscr{L}$. So the problem arises to describe in reasonable terms the convergence behavior of Fourier series in $\mathscr{L}$. Probably the best one can expect for decent functions is the existence of a bounded approximate identity, consisting of finite linear combinations of the $f_{j}$, for the algebra of all $g \in \mathscr{L}$ with $\lambda(g)=\sum \gamma, \lambda\left(f_{j}\right)$. The Fejer kernel does exactly this for the periodic functions.

Instead of studying individual functions it is more reasonable to investigate commutative selfadjoint closed subalgebras of $\mathscr{L}$. Recall that $\mathscr{Q}_{1}$ is dense in $L^{2}(G)$, see [9], thus, if $G$ is separable, $\mathscr{Q}_{1}$ contains orthonormal bases $\mathfrak{u}=\left\{u_{J}\right\}$ of $L^{2}(G)$. It is easy to see that for such a basis $\mathfrak{u}$ the set of elements $f$ in $\mathscr{L}$ having Fourier series $\sum \alpha_{j} u_{j} \circ u_{j}$ with $u_{j} \in \mathfrak{u}$ form a maximal selfadjoint commutative subalgebra $\mathscr{F}$ of $\mathscr{L}$. Actually, what is really needed for the maximality of $\mathscr{F}$ is the fact, that $\mathfrak{u}$ is a maximal orthonormal subset of $\mathscr{Q}_{1}$. 
Definition. Let $\mathfrak{u}=\left\{u_{j}\right\}$ be a subset of $\mathscr{Q}_{1}$, orthonormal and maximal in $\mathscr{Q}_{1}$ with respect to orthonormality and denote by $\mathscr{U}$ the family of all these $\mathfrak{u}$. For $\mathfrak{u} \in \mathscr{U}$ let $\mathscr{F}_{0}(\mathfrak{u})$ the closure in $\mathscr{L}$ of all finite linear combinations of the $e_{j}=u_{j} \circ u_{j}$, and $\mathscr{F}(\mathfrak{u})$ the family of all functions $f \in \mathscr{L}$, for which $\lambda(f)=\Sigma \zeta_{j} \lambda\left(e_{j}\right)$, i.e. which have Fourier series $\sum \alpha_{j} f_{j}$ with $f_{j} \in \mathscr{F}_{0}(\mathfrak{u})$.

Clearly $\mathscr{F}_{0}$ in the closed subalgebra generated by the $e_{j}$. Moreover:

Proposition 2. The correspondence $\mathfrak{u} \rightarrow \mathscr{F}(\mathfrak{u})$ is bijective between $\mathscr{U}$ and the set of all maximal selfadjoint commutative subalgebras of $\mathscr{L}$.

Proof. Let $\mathfrak{u} \in \mathscr{U}$ and assume that $h \in \mathscr{L}, h=h^{*}$, commutes with $\mathscr{F}(\mathfrak{u})$. If $h \sim \sum \alpha_{j} g_{j}$, then all $g_{j}$ commute with $\mathscr{F}(\mathfrak{u})$. This implies $g_{j}=g_{j}^{\prime}+g_{j}^{\prime \prime}$ with $g_{j}^{\prime} \in \mathscr{F}_{0}(\mathfrak{u}), g_{j}^{\prime \prime} e_{i}=0$ for all $e_{i}=u_{i} \circ u_{i} \in \mathscr{F}_{0}(\mathfrak{u})$. If $g_{j}^{\prime \prime} \neq 0$, then we could find some $v \in \mathscr{Q}_{1}$ with $|v|_{2}=1, v \circ v \leq g_{j}^{\prime \prime}$, hence $(v \circ v) e_{i}=0$, i.e. $v$ orthogonal to all $u_{i} \in \mathfrak{H}$, contradicting the maximality of $\mathfrak{u}$. It follows that all $g_{j}$ are $\mathscr{F}_{0}(\mathfrak{u})$ thus $h \in \mathscr{F}(u)$.

Now let $\mathscr{F}$ be a maximal selfadjoint commutative subalgebra of $L$. If $f \in \mathscr{F}$ has the Fourier series $\sum \alpha_{j} f_{j}$ then also all $f_{j}$ are in $\mathscr{F}$. Let $\gamma=\left\{g_{i}\right\}$ be a maximal family of pairwise orthogonal hermitian idempotents $g_{i} \in \mathscr{F}$ and minimal in $\mathscr{F}$. If $\sum \alpha_{j} f_{j} \sim f \in \mathscr{F}$, then for every $g_{i} \in \gamma$ and $f_{j}$ either $g_{i} \circ f_{j}=0$ or $g_{i} \circ f_{j}=g_{i}$, thus $g_{i} \circ f=\xi_{i} g_{i}$ for some $\xi_{i} \in \mathbf{C}$. Let $e$ be an idempotent of rank 1 with $e \leq g_{i}$, hence $e * g_{i}=g_{i} * e=e$. Then $e * f=$ $e * g_{i} * f=\xi_{i} e * g_{i}=\xi_{i} e=f * e$ for all $f \in \mathscr{F}$; hence $e \in \mathscr{F}, g_{i}=e$. It follows that all $g_{i} \in \gamma$ have rank 1. Moreover every hermitian idempotent in $\mathscr{F}$ is a sum of some $g_{i} \in \gamma$. If $g_{i}=u_{i} \circ u_{i}$, then $\left\{u_{i}\right\}$ is an orthonormal family of $L^{2}$, contained in $\mathscr{Q}_{1}$. If it would not be maximal we could find a $u \in \mathscr{Q}_{1},|u|_{2}=1$, orthogonal to all $u_{1}$. Then $e=u \circ u$ is nonzero and orthogonal on all $g_{i} \in \gamma$, hence $e * f=f * e=0$ for all $f \in \mathscr{F}$. This is impossible, because $\mathscr{F}$ is maximal. It follows that $\left\{u_{i}\right\}=u$ is in $\mathscr{U}$ and $\mathscr{F}=\mathscr{F}(\mathfrak{u})$.

The problem of convergence of Fourier series can now be stated in different degrees of strength:

$\left(\mathrm{C}_{1}\right) \mathscr{F}_{0}(\mathfrak{u})=\mathscr{F}(\mathfrak{u})$.

$\left(\mathrm{C}_{2}\right) \mathscr{F}_{0}(\mathfrak{u})$ has a bounded approximate identity for $\mathscr{F}(\mathfrak{u})$.

$\left(\mathrm{C}_{3}\right) \mathscr{F}_{0}(\mathfrak{u})$ contains a hermitian bounded approximate identity for $\mathscr{L}$.

REMARKs. (1) If $\left(\mathrm{C}_{3}\right)$ holds for some $\mathfrak{u}$, then (see [6] Satz 1$) 2$ has to have a bounded approximate identity. We will always assume that this is the case. 
(2) In the general case, $\mathscr{F}_{0}=\mathscr{F}_{0}(\mathfrak{u})$ is always a closed ideal in $\mathscr{F}=\mathscr{F}(\mathfrak{u})$ and the quotient $\mathscr{F} / \mathscr{F}_{0}$ is a radical algebra: Assume that $\varphi_{0}$ is a nonzero multiplicative bounded functional on $\mathscr{F}$. As $\mathscr{L}$, hence $\mathscr{F}$ is symmetric, $\varphi_{0}$ is hermitian, i.e. a character of $\mathscr{F}$. Again by symmetry $\varphi_{0}$ extends to a positive hermitian form $\varphi$ on $\mathscr{L}$. But these are continuous in the $C^{*}$-norm of $\mathscr{L}$, thus $\varphi_{0}\left(\mathscr{F}_{0}\right) \neq 0$, which proves our assertion.

(3) If $\mathscr{F}_{0}(\mathfrak{u})$ contains an approximate identity $\left\{h_{j}\right\}_{j}$, then we can of course assume that the $h_{j}$ are hermitian and contained in the linear span of the $g_{i}=u_{i} \circ u_{i}, u_{i} \in \mathfrak{u}$, i.e. that $h_{j}=\sum \eta_{j i} g_{i}$ with $\eta_{j i}=0$ for almost all $i$ for each $j$. These $\left\{h_{i}\right\}$ can be considered as special summation kernels, in both cases $\left(\mathrm{C}_{2}\right)$ and $\left(\mathrm{C}_{3}\right)$. The Fejer kernel in the case of the family $\mathfrak{u}=\left\{e^{2 \pi i j x}\right\}_{j \in \mathbf{Z}}$ and the algebra $L^{1}(\mathbf{T}, A(\mathbf{T})), \mathscr{F}_{0}(\mathfrak{u})=\mathscr{F}(\mathfrak{u})=L^{1}(\mathbf{T})$, is an example. In this case $\mathscr{F}(\mathfrak{u})$ satisfies $\left(\mathrm{C}_{3}\right)$.

For a fixed basis $\mathfrak{u}$ in $\mathscr{Q}_{1}$ let

$$
g_{j k}=u_{i} \circ u_{k}, \quad u_{i}, u_{k} \in \mathfrak{u},
$$

and denote by $\mathscr{E}(\mathfrak{u})$ the subalgebra of $\mathscr{E}$, generated by the $g_{j k}$, i.e. the set of all finite linear combinations of the $g_{j k}$.

Proposition 3. The algebra $\mathscr{E}(\mathfrak{u})$ is dense in $\mathscr{L}$ if and only if the linear span $\langle\mathfrak{u}\rangle$ of $\mathfrak{u}$ is dense in $\mathscr{Q}_{1}$.

Proof. Fix $u \in \mathfrak{u}$ with $|u|_{2}=1$ and set $p=u \circ u$, so that $p$ is a hermitian idempotent of rank 1 and for a closed left ideal $\mathscr{V} \subset \mathscr{L}$

$$
p * \mathscr{V}=u \circ \beta(\mathscr{V})
$$

where $\beta(\mathscr{V})$ is a closed subspace of $\mathscr{Q}_{1}$. Moreover $\beta: \mathscr{V} \rightarrow \beta(\mathscr{V})$ maps the lattice of closed left ideals in $\mathscr{L}$ bijectively onto the lattice of closed subspaces of $\mathscr{Q}_{1}$, see [9], Theorem 3 .

Now assume that $\mathscr{E}(\mathfrak{u})^{-}=\mathscr{L}$. Then

$$
u \circ \mathscr{Q}_{1}=p *(\mathscr{E}(\mathfrak{u}))^{-}=(p * \mathscr{E}(\mathfrak{u}))^{-}=(\mathfrak{u} \circ\langle\mathfrak{u}\rangle)^{-}=u \circ \overline{\langle\mathfrak{u}\rangle}
$$

and consequently $\langle\mathfrak{u}\rangle$ is dense in $\mathscr{Q}_{1}$.

On the other hand let $U=\overline{\langle\mathfrak{u t}} \neq \mathscr{Q}_{1}$. Then again by [9], Theorem 3, $\gamma(U)=(\mathscr{L} *(u \circ U))^{-} \neq \mathscr{L}$. As $\mathscr{E}(\mathfrak{u}) \subset \gamma(U)$ it follows that $\mathscr{E}(\mathfrak{u})^{-} \neq \mathscr{L}$.

COROLlaRY. If $\mathfrak{u}$ satisfies $\left(\mathrm{C}_{3}\right)$, then $\langle\mathfrak{u}\rangle$ is dense in $\mathscr{Q}_{1}$.

Proof. As remarked before, $\left(\mathrm{C}_{3}\right)$ implies that there exists a bounded approximate identity for $\mathscr{L}$ of finite sums of the $g_{i}=u_{i} \circ u_{i}$. Because all $g_{i}$ are in $\mathscr{E}(\mathfrak{u})$, it follows that $\mathscr{E}(\mathfrak{t})^{-}=\mathscr{L}$, hence $\overline{\langle\mathfrak{t}\rangle}=\mathscr{Q}_{1}$. 
It is easy to construct bases $\mathfrak{u}$ in $\mathscr{Q}_{1}$ for which $\langle\mathfrak{u}\rangle$ is not dense, one only has to take a closed proper subspace $U \subset \mathscr{Q}_{1}$, which is dense in $L^{2}(G)$, e.g. all $a \in \mathscr{Q}_{1}$ which vanish on a fixed closed subset in $G$ of measure 0 . Then clearly $U$ contains a basis $\mathfrak{u}$ for $L^{2}$ with $\overline{\langle\mathfrak{u}\rangle} \subset U$, thus $\overline{\langle\mathfrak{u}\rangle} \neq \mathscr{Q}_{1}$.

On the other hand if $\langle\mathfrak{u}\rangle$ is dense in $\mathscr{Q}_{1}$, then $\mathscr{E}(\mathfrak{u})$ is dense in $\mathscr{L}$ and consequently there exists a bounded approximate identity $\left\{e_{i}\right\}$ of the form $e_{i}=\sum \gamma_{j k}^{i} g_{j k}$ with finite hermitian matrices $\gamma^{i}=\left(\gamma_{j k}^{i}\right)_{j k} \cdot\left(\mathrm{C}_{3}\right)$ means that we can choose real diagonal matrices for the $\gamma^{i}$.

Now we consider a connected compact abelian group $K$ and assume that $K$ acts continuously on $\mathscr{L}$, i.e. we have a strongly continuous homeomorphism from $K$ into the group of isometric $*$-automorphism of $\mathscr{L}$. We denote this action by $f \rightarrow f^{k}$ for $f \in \mathscr{L}, k \in K$. Then the representations $f \rightarrow \lambda\left(f^{k}\right)$ for fixed $k \in K$ are equivalent to $\lambda$, hence there exists a projective unitary representation $U: k \rightarrow U_{k}$ of $K$ in $L^{2}(G)$ with

$$
\lambda\left(f^{k}\right)=U_{k}^{*} \lambda(f) U_{k}
$$

for all $f \in \mathscr{L}, k \in K$ and as $K$ is connected, we can and will assume that $U$ is an ordinary continuous unitary representation of $K$. The representation $U$ is uniquely defined by the action of $K$ on $\mathscr{L}$, up to a multiplication by a unitary character of $K$.

Let $a, b, c$ be elements in $\mathscr{Q}_{1}$. Then $a \circ b$ is a rank 1 element in $\mathscr{L}$, consequently also $(a \circ b)^{k}$ for $k \in K$, thus $(a \circ b)^{k}=a_{k} \circ b_{k}$ for some $a_{k}$, $b_{k} \in \mathscr{Q}_{1}$. Now $\lambda(a \circ b) c=(c \mid b) a$, hence

$$
\begin{aligned}
\lambda\left(a_{k} \circ b_{k}\right) U_{k}^{*} c & =U_{k}^{*} \lambda(a \circ b) U_{k} \cdot U_{k}^{*} c=U_{k}^{*}(\lambda(a \circ b) c) \\
& =U_{k}^{*}((c \mid b) a)=(c \mid b) U_{k}^{*} a=\left(U_{k}^{*} c \mid b_{k}\right) a_{k}
\end{aligned}
$$

and $(c \mid b) U_{k}^{*} a=\left(c \mid U_{k} b_{k}\right) a_{k}$. Taking $b=c,|b|_{2}=|a|_{2}=1$, we see that $U_{k}^{*} a=\left(b \mid U_{k} b_{k}\right) a_{k}$, thus, because also $\left|a_{k}\right|_{2}=1, U_{k} b_{k}=\zeta b, a_{k}=\bar{\zeta} U_{k}^{*} a$ with $\zeta \in \mathbf{C},|\zeta|=1$. It follows that

$$
(a \circ b)^{k}=\left(U_{k}^{*} a\right) \circ\left(U_{k}^{*} b\right)
$$

in particular $\mathscr{Q}_{1}$ is $U$-invariant.

Proposition 4. The restriction of $U$ onto $\mathscr{Q}_{1}$ acts continuously on the Banach space $\mathscr{Q}_{1}$, i.e. $\mathscr{Q}_{1}$ is a Banach $K$-module, in particular

$$
\mathscr{Q}_{1}=\left(\sum_{\chi \in \hat{K}}^{\oplus} \mathscr{Q}_{1}^{\chi}\right)^{-}
$$


i.e. $\mathscr{Q}_{1}$ is the closure of the direct sum of the eigenspaces

$$
\mathscr{Q}_{1}^{\chi}=\left\{a \in \mathscr{Q}_{1} ; U_{k} a=\chi(k) a\right\}
$$

of the characters $\chi$ of $K$. We can choose $U$ so that for the trivial character $\varepsilon$ we have $\mathscr{Q}_{1}^{\varepsilon} \neq 0$.

Proof. We have for $\mathscr{L}$ a corresponding decomposition into the subspaces $\mathscr{L}^{x}$ and because $K$ acts by *-automorphisms it follows that

$$
\mathscr{L}^{\chi} * \mathscr{L}^{\Psi} \subset \mathscr{L}^{\chi \Psi}, \quad\left(\mathscr{L}^{\chi}\right)^{*}=\mathscr{L}^{\bar{\chi}}
$$

hence $\mathscr{L}^{\chi} *\left(\mathscr{L}^{\chi}\right)^{*} \subset \mathscr{L}^{\varepsilon}$ for every $\chi \in \hat{K}$. Thus $\mathscr{L}^{\varepsilon} \neq 0$. If $f=f^{*} \in \mathscr{L}^{\varepsilon}$, $f \sim \sum \alpha_{j} f_{j}$ with hermitian idempotents $f_{j}$, then also the $f_{j}$ are invariant. Now let $f$ be an invariant hermitian idempotent of minimal rank $m$. Then $f * \mathscr{L} * f=\mathscr{L}_{f}$ is an invariant subalgebra of $\mathscr{L}$, isomorphic to the $m \times m$ matrixalgebra over $\mathbf{C}$. As before, the invariant elements form a $*$-subalgebra $\mathscr{L}_{f}^{\varepsilon} \neq 0$. Then the minimality of $m$ implies $\mathscr{L}_{f}^{\varepsilon}=\mathscr{L}_{f} \cong$ C, i.e. $m=1$.

Now let $n \in \mathscr{Q}_{1}$ be such that $u \circ u$ is an invariant idempotent. Then, as we have seen

$$
U_{k}^{*} u=\omega_{k} u
$$

with $\omega_{k} \in \mathbf{T}$, i.e. $u$ is an eigenvector for $U$ and $\omega: k \rightarrow \omega_{k}$ a continuous character. But then also $U^{\prime}: k \rightarrow \omega(k) U_{k}$ implements the action of $K$ on $\mathscr{L}$, so we can assume that $U^{\prime}=U, \omega=\varepsilon$ and $u \in \mathscr{Q}_{1}^{\varepsilon}$. Finally for arbitrary $a \in \mathscr{Q}_{1}$ and the invariant $u$ with $|u|_{2}=1$ we have a constant $\gamma>0$ with $\gamma\|v\|=|v \circ u|_{1}$, hence

$$
\begin{aligned}
\gamma\left\|U_{k}^{*} a-a\right\| & =\left|\left(U_{k}^{*} a\right) \circ u-a \circ u\right|_{1}=\left|U_{k}^{*} a \circ U_{k}^{*} u-a \circ u\right|_{1} \\
& =\left|(a \circ u)^{k}-(a \circ u)\right|_{1}
\end{aligned}
$$

which shows the continuity of $k \rightarrow U_{k}^{*} a$ in the $\mathscr{Q}_{1}$-norm.

For $a \in \mathscr{Q}_{1}^{\varphi}, b \in \mathscr{Q}_{1}^{\Psi}$ and $k \in K$ we obtain from (3):

$$
(a \circ b)^{k}=\overline{\varphi(k)} \Psi(k)(a \circ b)=\left(\varphi^{-1} \Psi\right)(k)(a \circ b),
$$

hence Proposition 4 has the

COROLlary. The eigenspaces

$$
\mathscr{L}^{x}=\left\{f \in \mathscr{L} ; f^{k}=\chi(k) f, k \in K\right\}
$$

are the closures of the linear span of all functions $a \circ b$ with $a \in \mathscr{Q}_{1}^{\varphi}$, $b \in \mathscr{Q}_{1}^{\varphi \chi}, \varphi \in \hat{K}$, in short

$$
\mathscr{L}^{\chi}=\left(\sum_{\varphi \in \hat{K}} \mathscr{Q}_{1}^{\varphi} \circ \mathscr{Q}_{1}^{\varphi \chi}\right)^{-},
$$


in particular, the subalgebra $\mathscr{L}^{\varepsilon}$ is the closure of the direct sum of the simple subalgebras

$$
\mathscr{L}_{\varphi}^{\varepsilon}=\left\{\sum a \circ b ; a, b \in \mathscr{Q}_{1}^{\varphi}\right\}^{-}
$$

Our next proposition holds also without the assumption that the compact group $K$ is commutative.

Proposition 5. $\mathscr{L}$ contains a bounded approximate unit $\left\{h_{\mu}\right\}_{\mu}$ of $K$-invariant hermitian elements $h_{\mu}$ of finite rank.

Proof. Let $\left\{g_{\mu}\right\}$ be an approximate identity for $\mathscr{L}$, bounded by $C$, i.e. $\left|g_{\mu}\right| \leqq C$ for all $\mu$. For $f \in \mathscr{L}$ let $f^{\#} \in \mathscr{L}^{\varepsilon}$ be defined by

$$
f^{\#}=\int_{K} f^{k} d k,
$$

hence $f \rightarrow f^{\#}$ projects $\mathscr{L}$ onto $\mathscr{L}^{\varepsilon}$ and $\left|f^{\#}\right|_{1} \leqq|f|_{1}$. We claim that $\left\{g_{\mu}^{\#}\right\}$ is an approximate identity, also bounded by $C$. As the last assertion is

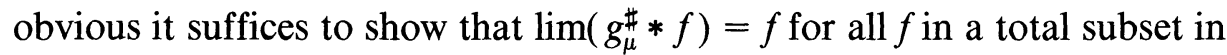
$\mathscr{L}$, e.g. for all $f \in \bigcup_{\Psi \in \hat{K}} \mathscr{L}^{\Psi}$. So let $f \in \mathscr{L}^{\Psi}$, i.e. $f^{k}=\Psi(k) f$. Then

$$
\begin{aligned}
\left|g_{\mu}^{\#} * f-f\right|_{1} & =\left|\int_{K}\left(g_{\mu}^{k} * f-f\right) d k\right|_{1}=\left|\int\left(g_{\mu} * f^{k^{-1}}-f^{k^{-1}}\right)^{k} d k\right|_{1} \\
& =\left|\int \overline{\Psi(k)}\left(g_{\mu} * f-f\right)^{k} d k\right|_{1}\left|g_{\mu} * f-f\right|_{1},
\end{aligned}
$$

hence $\lim _{\mu}\left(g_{\mu}^{\#} * f\right)=f$ und $\left\{g_{\mu}^{\#}\right\}_{\mu}$ is a bounded approximate identity in $\mathscr{L}^{\varepsilon}$.

For every $\sigma$ with $0<\sigma<1$ and every $\mu$ we can choose a finite rank element $j_{\mu, \sigma}$ in $\mathscr{L}^{\varepsilon}$ with $\left|g_{\mu}^{\#}-j_{\mu, \sigma}\right|_{1}<\sigma$. If we order the pairs $(\mu, \sigma)$ by $(\mu, \sigma) \geq(\nu, \tau)$ if $\mu \geq \nu, \sigma \leq \tau$, then also $\left\{j_{\mu, \sigma}\right\}_{\mu, \sigma}$ is a bounded approximate identity and finally the family $\left\{h_{\mu, \sigma}\right\}_{\mu, \sigma}$ with $h_{\mu, \sigma}=\frac{1}{2}\left(j_{\mu, \sigma}+j_{\mu, \sigma}^{*}\right)$ has all the properties claimed in Proposition 3.

It is clear that the algebra $\mathscr{L}^{\varepsilon}$ of invariant elements is commutative if and only if all $\mathscr{L}_{\varphi}^{\varepsilon}, \varphi \in \hat{K}$, defined by (4), are zero- or one-dimensional, which, in turn, is equivalent to the fact that $\operatorname{dim} \mathscr{Q}_{1}^{\varphi} \leqq 1$ for all $\varphi \in \hat{K}$.

Definition. We say that $K$ acts radially on $\mathscr{L}$, if $\operatorname{dim} \mathscr{Q}_{1}^{\varphi} \leqq 1$ for all $\varphi \in \hat{K}$. A function $f \in \mathscr{L}$ is called radial (with respect to $K$ ) if it is invariant under $K$, i.e. if $f \in \mathscr{L}^{\varepsilon}$.

So $K$ acts radially on $\mathscr{L}$ if and only if the multiplicities of the implementing representation $U$ of $K$ in $L^{2}(G)$ are all at most 1. From our 
previous results we conclude:

THEOREM 1. If the commutative compact group $K$ acts radially on $\mathscr{L}$ then the radial functions form a maximal commutative subalgebra, i.e. $\mathscr{L}^{\varepsilon}=\mathscr{F}(\mathfrak{u})$, where $\mathfrak{u}=\left\{u_{\chi}\right\}$ and the $u_{\chi}$ are eigenfunctions of the representation $U$ of $K$ in $\mathscr{Q}_{1}$ for the character $\chi$ of $K . \mathscr{L}^{\varepsilon}$ satisfies condition $\left(\mathrm{C}_{3}\right)$, i.e. $\mathscr{F}_{0}(\mathfrak{u})$ contains a hermitian bounded approximate identity for $\mathscr{L}$.

REMARKS. In the radial case the $u_{\chi}$ in $\mathfrak{u}$ generate the non-zero primary eigenspaces of the $K$-module $\mathscr{Q}_{1}$, i.e. $\mathscr{Q}_{1}^{\chi}=\mathbf{C} \cdot u_{\chi}$, if $\mathscr{Q}_{1}^{\chi} \neq 0$. Of course it is possible that $\mathscr{Q}_{1}^{\chi}=0$ for some $\chi \in \hat{K}$, moreover, while the family $\left\{\mathscr{Q}_{1}^{\chi} ; u_{\chi}\right.$ $\neq 0, \chi \in \hat{K}\}$ is uniquely defined by the action of $K$ on $\mathscr{L}$, the actual application $\chi \rightarrow 2_{1}^{\chi}$ depends also on the choice of $U$.

As a consequence of the theorem we see that for radial functions in $\mathscr{L}^{\varepsilon}$ there exists a summation method: Let us assume that $\mathfrak{u}=\left\{u_{j}\right\}$ is countable, e.g. $\hat{K}=\left\{\chi_{j}\right\}, u_{j}=u_{\chi_{j}}, j \in \mathbf{N}$. Then the approximate unit $\left\{h_{n}\right\}_{n}$ of radial finite rank functions $h_{n}$ can be taken countable. With $e_{j}=u_{J} \circ u_{j}$ we have

$$
h_{n}=\sum_{j=0}^{r_{n}} s_{n j} e_{j}
$$

with positive real $s_{n j}$. Now let $f \in \mathscr{L}^{\varepsilon}$ have the Fourier series $\sum \alpha_{j} e_{j}$. Then $h_{n} * f=\sum_{j=0}^{r_{n}} s_{n j} \alpha_{j} e_{j}$ and we have

$$
\lim _{n \rightarrow \infty}\left(\sum_{j=0}^{r_{n}} s_{n j} \alpha_{j} e_{j}\right)=f \text { in } \mathscr{L} .
$$

Our first example will show that the Fejer kernel for periodic $L^{1}$-functions is a special case of such an approximate identity.

Assume that $K$ is a central subgroup of $G$. Then we define the action of $K$ on $\mathscr{L}$ by

$$
f^{k}(x)(y)=f(x)^{k}(y)=f(x, k y),
$$

i.e. we extend the natural action of $K$ on $\mathscr{Q}$, defined by $q \rightarrow q^{k}, q^{k}(y)=$ $q(k y)=q(y k)$ for $q \in \mathscr{Q}$, to $\mathscr{L}$. It is clear that $\mathscr{L}$ is a $K$-algebra with this action. Moreover it follows immediately from (5), that $f \in \mathscr{L}$ is invariant if and only if all values $f(x)$ as functions in $\mathscr{Q}$ depend only on the cosets $\dot{y}=y K \in G / K$. Let $\dot{\mathscr{Q}}$ be the subalgebra of all $K$-invariant functions in 2 . Then we can consider $\dot{\mathscr{Q}}$ canonically as a subalgebra of $C_{\infty}(G / K)$ and we have

$$
\mathscr{L}^{\varepsilon}=L^{1}(G, \dot{\mathscr{Q}}) \subset \mathscr{L}
$$


Algebras of this type have been studied in [9]. Clearly the action of $K$ on $\mathscr{L}$ is radially if and only if $G=K$. In this case $\mathscr{L}^{\varepsilon}=L^{1}(K)$ and the Fourier series for $f \in \mathscr{L}^{\varepsilon}$ is just the usual one: $\sum_{\hat{K}} \hat{f}(\chi) \cdot \chi$.

Our next example is more important and more involved. Let $\mathbf{H}_{n}$ be the $(2 n+1)$-dimensional Heisenberg group. As usually we can write $\mathbf{H}_{n}=\mathbf{R}^{2 n} \times \mathbf{R}$ with product

$$
(x, s)(y, t)=(x+y, s+t+\langle x, y\rangle)
$$

with a bilinear form $\langle x, y\rangle$ on $\mathbf{R}^{2 n}$ for which the difference $\langle x, y\rangle$ $-\langle y, x\rangle$ is nondegenerate. Different forms $\langle x, y\rangle$ correspond to different sections $\mathbf{R}^{2 n}=\mathbf{H}_{n} / Z \rightarrow \mathbf{H}_{n}, Z \cong \mathbf{R}$ the center of $\mathbf{H}_{n}$. Most frequently used are the isotropic and the polarized sections with the corresponding form, see [2]: The isotropic section is the natural one in the complex parametrisation of $\mathbf{H}_{n}$. We identify $\mathbf{R}^{2 n}$ with $\mathbf{C}^{n}$ and denote the usual positive definite inner product by $(x \mid y)$, i.e. $(x \mid y)=\sum_{1}^{n} x_{j} \bar{y}_{j}$. Then the isotropic form is defined by

$$
\langle x, y\rangle_{i}=\frac{1}{2} \operatorname{Im}(x \mid y) .
$$

The polarized form comes from the usual representation of $\mathbf{H}_{n}$ by lower triangular real $n \times n$-matrices and is defined by

$$
\langle x, y\rangle_{p}=x^{\prime \prime} y^{\prime}
$$

if $x=\left(x^{\prime}, x^{\prime \prime}\right), x^{\prime}, x^{\prime \prime} \in \mathbf{R}^{n}$, similarly $y=\left(y^{\prime}, y^{\prime \prime}\right)$ and $u v=\sum_{j=1}^{n} u_{j} v_{j}$ for $u, v \in \mathbf{R}^{n}$. If

$$
\beta(x)=\beta\left(x^{\prime}, x^{\prime \prime}\right)=\frac{1}{2} x^{\prime} x^{\prime \prime}
$$

then the two forms are related by

$$
\langle x, y\rangle_{p}-\langle x, y\rangle_{i}=\beta(x+y)-\beta(x)-\beta(y) .
$$

For the rest of the paper let $K=\mathbf{T}^{n}$. Then $K$ acts by multiplication isometrically on $\mathbf{C}^{n}$ and leaves $(x \mid y)$ fixed. Using $\langle,\rangle_{i}$ in (6) for defining the product in $\mathbf{H}_{n}$ we see that

$$
\zeta(x, s)=(\zeta x, s), \quad \zeta \in K,(x, s) \in \mathbf{C}^{n} \times \mathbf{R}
$$

defines an automorphism $\alpha_{\zeta}$ of $\mathbf{H}_{n}$, leaving the center elementwise fixed. It is clear that $\alpha: \zeta \rightarrow \alpha_{\zeta}$ has all the necessary continuity properties, hence $\alpha$ acts continuously on $L^{1}\left(\mathbf{H}_{n}\right)$ and likewise on all infinite-dimensional primitive quotients $L_{\lambda}^{1}\left(\mathbf{H}_{n}\right)=L^{1}\left(\mathbf{H}_{n}\right) / \operatorname{ker} \pi_{\lambda}$, where $\pi_{\lambda}$ is the irreducible representation of $L^{1}$ in $L^{2}\left(\mathbf{R}^{n}\right)$, corresponding to $\lambda \in \mathbf{R}^{\times}$. Now all these quotients are isomorphic to

$$
\Gamma\left(\mathbf{R}^{n}\right)=L^{1}\left(\mathbf{R}^{n}, A\left(\mathbf{R}^{n}\right)\right),
$$


where $A\left(\mathbf{R}^{n}\right)=\mathscr{F}\left(L^{1}\left(\mathbf{R}^{n}\right)\right)$ is the Fourier algebra of $\mathbf{R}^{n}$ and $\mathbf{R}^{n}$ acts on $A\left(\mathbf{R}^{n}\right)$ by translation, see [7]. The isomorphism is established by using the polarized form for the product and applying Fourier transformation to $x^{\prime \prime}$. Unfortunately there is no simple formula for the action of $K$ on $L^{1}\left(\mathbf{H}_{n}\right)$ in terms of the polarized form, thus it seems not to be possible to give a short explicit expression for the image $f^{\zeta}$ of a function $f \in \Gamma\left(\mathbf{R}^{n}\right)$ for $\zeta \in K$. But using the fact that

$$
\beta(x, y)=\frac{1}{2} x y=\frac{1}{4} \operatorname{Im}\left(\sum_{j}\left(x_{j}+i y_{j}\right)^{2}\right)
$$

and the relationship between the parametrisations of $\mathbf{H}_{n}$ corresponding to $\langle,\rangle_{i}$ and $\langle,\rangle_{p}$ one sees that the formula for the action of $K$ with respect to the polarized form is given by

$$
\zeta(x, s)=\left(\zeta x, s+\frac{1}{4} \operatorname{Im}\left((\zeta x)^{2}-x^{2} \mid 1\right)\right)
$$

where of course $\mathbf{C}^{n}$ is considered as the direct sum algebra $\mathbf{C} \oplus \cdots \oplus \mathbf{C}$, $n$ times, $\mathbf{1}=(1,1, \ldots, 1)$, and $x=\left(x^{\prime}, x^{\prime \prime}\right) \in \mathbf{R}^{2 n}$ is identified with $x=x^{\prime}$ $+i x^{\prime \prime} \in \mathbf{C}^{n}$.

The primitive quotient $L_{\lambda}^{1}\left(\mathbf{H}_{n}\right)=L_{\lambda}^{1}$ is obtained by partial Fourier transform of $f \in L^{1}\left(\mathbf{H}_{n}\right)$ :

$$
f_{\lambda}(x)=\int f(x, s) e^{-2 \pi i \lambda s} d s .
$$

Thus the action of $K$ on $L_{\lambda}^{1}$ is given by

$$
f_{\lambda}^{\zeta}(x)=\left(f_{\lambda}\right)^{\zeta}(x)=e^{\pi \lambda i \operatorname{Im}\left((\zeta x)^{2}-x^{2} \mid 1\right) / 2} f_{\lambda}(\zeta x)
$$

and $g \in L_{\lambda}^{1}$ is $K$-invariant if and only if

$$
e^{\pi \lambda i \operatorname{Im}\left(x^{2} \mid 1\right) / 2} g(x)=e^{\pi \lambda i \operatorname{Im}\left((\zeta x)^{2} \mid 1\right) / 2} g(\zeta x),
$$

i.e. if the left-hand side function is (poly-) radial on $\mathbf{C}^{n}$ in the ordinary sense. Thus, writing $|x|=\left(\left|x_{1}\right|, \ldots,\left|x_{n}\right|\right)$ for $x=\left(x_{1}, \ldots, x_{n}\right) \in \mathbf{C}^{n}$, the general invariant function $g \in L_{\lambda}^{1}$ is given by

$$
g(x)=e^{-\pi \lambda i \operatorname{Im}\left(x^{2} \mid 1\right) / 2} g_{0}(|x|)
$$

with a function $g_{0} \in L^{1}\left(\mathbf{C}^{n}\right)$, depending only on $|x|$.

Now we want to switch from $L_{\lambda}^{1}$ to $\Gamma_{\lambda}\left(\mathbf{R}^{n}\right)$, with $\Gamma_{\lambda}\left(\mathbf{R}^{n}\right)=$ $L^{1}\left(\mathbf{R}^{n}, A\left(\mathbf{R}^{n}\right)\right)$, but action $u^{x}(y)=u(y+\lambda x)$ for $x \in \mathbf{R}^{n}$. So $\Gamma_{1}\left(\mathbf{R}^{n}\right)$ is our "old" $\Gamma\left(\mathbf{R}^{n}\right)$, corresponding to $\lambda=1$. Therefore we change the notation and write $(x, y)$ instead of $x=\left(x^{\prime}, x^{\prime \prime}\right)$, resp. $x=x^{\prime}+i x^{\prime \prime}$. Then the isomorphism from $L_{\lambda}^{1}$ onto $\Gamma_{\lambda}\left(\mathbf{R}^{n}\right)$ is a Fourier transform with respect to 
$y$, furthermore, $\operatorname{Im}\left(x^{2} \mid 1\right)$ becomes $2 x y=2 \sum x_{j} y_{j}$ and (9) implies that

$$
g(x, y)=\int_{\mathbf{R}^{n}} h\left(x^{2}+t^{2}\right) e^{-2 \pi i(\lambda x / 2+y) t} d t
$$

defines a radial function in $\Gamma_{\lambda}\left(\mathbf{R}^{n}\right)$, whenever $h$ is a sufficiently well behaved function on $\left(\mathbf{R}^{+}\right)^{n}$ with $x^{2}+t^{2}=\left(x_{1}^{2}+t_{1}^{2}, \ldots, x_{n}^{2}+t_{n}^{2}\right) \in\left(\mathbf{R}^{+}\right)^{n}$. It is easy to see that $g$ is hermitian if and only if $h$ is real valued. To keep matters simple we restrict our computations to the case $\lambda=1$, hence $\Gamma_{\lambda}=\Gamma$. Taking $h\left(x^{2}+t^{2}\right)=e^{-\pi\left(x^{2}+t^{2}\right) / 2}$ in (10) we obtain a radial function $f$ with

$$
f(x-y, y)=e^{-\pi(x-y)^{2} / 2} \int e^{-\pi t^{2} / 2-\pi i(x+y) t} d t .
$$

Substituting $\sqrt{2} t$ for $t$ and observing that the Gauss-function $e^{-\pi t^{2}}$ is fixed under Fourier transform we obtain

$$
f(x-y, y)=2^{n / 2} e^{-\pi(x-y)^{2} / 2} e^{-\pi(x+y)^{2} / 2}=2^{n / 2} e^{-\pi x^{2}} e^{-\pi y^{2}} .
$$

Hence we see that for the normalized Gauss-function

$$
u_{0}(x)=2^{-n / 4} e^{-\pi x^{2}}
$$

the minimal hermitian idempotent

$$
p_{0}=u_{0} \circ u_{0}
$$

is $K$-invariant, in particular we have $u_{0} \in A\left(\mathbf{R}^{n}\right)_{1}^{\varepsilon}$, if the representation $U$ is properly normalized, see Proposition 4.

Next we will compute the action of $K$ on the Lie algebra $\mathfrak{h}_{n}$ of $\mathbf{H}_{n}$, or more exactly on the image of $\mathfrak{h}_{n}$ under the infinitesimal representation of $\mathfrak{h}_{n}$ in $L^{2}\left(\mathbf{R}^{n}\right)$ corresponding to $\pi_{1}$. In coincidence with our previous notations we will write $\lambda$ for the representation $\pi_{1}$ of $\Gamma\left(\mathbf{R}^{n}\right)$ and also for the corresponding representations of $\mathbf{H}_{n}$ and $\mathfrak{h}_{n}$. Then for $(x, y, t) \in \mathbf{H}_{n}$ the unitary operator $\lambda(x, y, t)$ in $L^{2}\left(\mathbf{R}^{n}\right)$ is given by

$$
(\lambda(x, y, t) \xi)(w)=e^{-2 \pi i(t+y(w-x))} \xi(w-x)
$$

for $\xi \in L^{2}\left(\mathbf{R}^{n}\right)$. Let $\left\{X_{j}, Y_{k}, Z\right\}$ be the usual basis of $\mathfrak{h}_{n}$, corresponding to the one-parameter subgroups $\left\{\left(t e_{j}, 0,0\right),\left(0, t e_{k}, 0\right),(0,0, t)\right\}, t \in \mathbf{R}, e_{j}$ the $j$ th unit vector in $\mathbf{R}^{n}$. As is well known one obtains the images

$$
A_{j}=\lambda\left(X_{j}\right), \quad B_{k}=\lambda\left(Y_{k}\right), \quad C=\lambda(Z)
$$

by differentiating (11):

$$
A_{J}=\frac{\partial}{\partial x_{j}}, \quad B_{k}=-2 \pi i M_{k}, \quad C=-2 \pi i 1
$$


with $\left(M_{k} \xi\right)(x)=x_{k} \xi(x), x \in \mathbf{R}^{n}$. In order to determine the images $X_{j}^{\zeta}$ etc. for $\zeta \in K$ one has to compute the images of the corresponding one-parameter subgroups. Putting $\zeta=c+i$ for $\zeta \in K$ (hence $c=$ $\cos (\arg \zeta), s=\sin (\arg \zeta))$ one obtains

$$
\begin{aligned}
& \zeta\left(t e_{j}, 0,0\right)=\left(t c_{j} e_{j}, t s_{j} e_{j}, \frac{1}{2} t^{2} c_{j} s_{j}\right) \\
& \zeta\left(0, t e_{j}, 0\right)=\left(-t s_{j} e_{j}, t c_{j} e_{j},-\frac{1}{2} t^{2} c_{j} s_{j}\right) .
\end{aligned}
$$

Inserting this in (11) and differentiating yields

$$
\begin{aligned}
& A_{j}^{\zeta}=c_{j} A_{j}+s_{j} B_{j}=\lambda\left(X_{j}^{\zeta}\right), \\
& B_{j}^{\zeta}=-s_{j} A_{j}+c_{j} B_{j}=\lambda\left(Y_{j}^{\zeta}\right) ;
\end{aligned}
$$

hence for

$$
C_{j}=A_{j}+i B_{j}=-\frac{\partial}{\partial x_{j}}+2 \pi M_{j}
$$

we have, with $\bar{C}_{j}=A_{j}-i B_{j}$,

$$
C_{j}^{\zeta}=\bar{\zeta}_{j} C_{j}, \quad \bar{C}_{j}^{\zeta}=\zeta_{j} \bar{C}_{j} .
$$

For a multi-index $q=\left(q_{1}, q_{2}, \ldots, q_{n}\right)$ with $q_{j} \in \mathbf{N}(0 \in \mathbf{N}$ !) we set

$$
C^{q}=\prod C_{j}^{q_{j}}, \quad \zeta^{q}=\prod \zeta_{j}^{q_{j}}, \quad x^{q}=\prod x_{j}^{q_{j}},
$$

hence $C^{q}$ is a differential operator of degree $|q|=\sum q_{j}$ and $\zeta^{q} \in \mathbf{T}, x^{q}$ a monomial of degree $|q|$. Applying $C^{q}$ to $u_{0}$ and multiplying with a factor $\gamma_{q}$ we obtain the hermitian functions

$$
u_{q}=\gamma_{q} C^{q} u_{0}=h_{q}(x) e^{-\pi x^{2}}
$$

with $\left|u_{q}\right|_{2}=1, h_{q}$ the $q$ th hermitian polynomial in $x=\left(x_{1}, \ldots, x_{n}\right)$. From

$$
U_{\xi}^{*} u_{q}=\gamma_{q}\left(C^{q}\right)^{\xi} U_{\zeta}^{*} u_{0}=\gamma_{q} \xi^{q} C^{q} u_{0}=\zeta^{q} u_{q}
$$

we see that $u_{q}$ is an eigenfunction of $U$ for the eigenvalue $\zeta^{q}$. As all $u_{q}$ are contained in $A\left(\mathbf{R}^{n}\right)_{1}$ (see e.g. [12]) and $\left\{u_{q}\right\}_{q}=\mathfrak{u}$ is an orthonormal base of $L^{2}\left(\mathbf{R}^{n}\right)$, we see that $u_{q}$ generates the eigenspace $A\left(\mathbf{R}^{n}\right)_{1}^{q}$ for the character $\zeta \rightarrow \zeta^{q}$ of $K$. Consequently we have proved the following

THEOREM 2. The group $K=\mathbf{T}^{n}$ acts radially on $\Gamma\left(\mathbf{R}^{n}\right)$, in particular

$$
A\left(\mathbf{R}^{n}\right)_{1}=\left(\sum_{q \in \mathbf{N}^{n}}^{\oplus}\left(u_{q}\right)\right)^{-}
$$

where $u_{q}$ is the qth Hermite function on $\mathbf{R}^{n}$. 
COROLlARY. The radial functions $\Gamma^{\#}$ form a maximal commutative *-subalgebra of $\Gamma\left(\mathbf{R}^{n}\right)$, containing a bounded approximate identity of finite rank elements.

As a fairly immediate consequence of the corollary one can prove that also the subalgebra $L_{K}^{1}\left(\mathbf{H}^{n}\right)$ of radial functions in $L^{1}\left(\mathbf{H}^{n}\right)$ is a maximal commutative *-subalgebra, because a subalgebra $\mathscr{B} \subset L^{1}\left(\mathbf{H}^{n}\right)$ is commutative if and only if all images $\mathscr{B}_{\lambda}$ of $\mathscr{B}$ in the primitive quotients $L_{\lambda}^{1}$ are commutative. More generally one can consider the situation in which $K=\mathbf{T}^{n}$ acts on a connected nilpotent Lie group $G$. Then again $K$ acts on $L^{1}(G)$ and the functions invariant under this action form a subalgebra $L_{K}^{1}(G)$.

Problem. Find necessary and sufficient conditions for the commutativity of $L_{K}^{1}(G)$. We will solve this problem for nilpotent groups. We assume first that $G$ is of class 2 .

The first step is a reduction: Instead of studying $G$ we consider the Lie algebra $\mathfrak{g}$ of $G$ and assume that $K$ acts on $\mathfrak{g}$. Let $z$ be the center of $\mathfrak{g}$ and $\mathfrak{g}^{\prime}=[\mathfrak{g}, \mathfrak{g}]$ the derived algebra. Clearly $z$ and $\mathfrak{g}^{\prime}$ are invariant and $\mathfrak{g}^{\prime} \subset \mathfrak{z}$, thus one can directly decompose $\mathfrak{g}$ into invariant subspaces:

$$
g=e \oplus g^{\prime} \oplus a, \quad z=g^{\prime} \oplus a
$$

It follows that $\mathfrak{g}_{1}=e \oplus \mathfrak{g}^{\prime}$ is an invariant subalgebra with $\mathfrak{g}_{1}^{\prime}=\mathfrak{g}^{\prime}=z_{1}=$ center of $\mathfrak{g}_{1}$, and $\mathfrak{g}$ is the algebra-direct sum of $\mathfrak{g}_{1}$ and the commutative algebra a. Correspondingly $G=G_{1} \times A$ with $A \cong \mathbf{R}^{a}, L^{1}(G)=L^{1}\left(G_{1}\right) \hat{\otimes}$ $L^{1}(A)$. Now one can prove that $L_{K}^{1}(G)$ is commutative if and only if $L_{K}^{1}\left(G_{1}\right)$ is commutative. This reduces the problem to the case

$$
\mathfrak{g}^{\prime}=\mathfrak{z}
$$

resp. $G^{\prime}=Z$ for the group $G$. So from now on we will assume that $G$ is connected, simply connected with algebra $g$ and such that (12) is satisfied. Under these assumptions we will prove

THEOREM 3. The subalgebra $L_{K}^{1}(G)$ of invariant functions is commutative if and only if the following conditions are fulfilled:

(i) $K$ acts trivially on $z$ (resp. $Z$ );

(ii) $n(=\operatorname{dim} K)=\frac{1}{2} \operatorname{dim}(\mathrm{g} / \mathrm{z})$.

Under these conditions $G$ is a quotient of the direct product $\mathbf{H}^{1} \times \mathbf{H}^{1} \times$ $\cdots \times \mathbf{H}^{1}=\left(\mathbf{H}^{1}\right)^{\times n}$, with the obvious action of $K=\mathbf{T}^{n}$ on $\left(\mathbf{H}^{1}\right)^{\times n}$. 
We start the proof with some simple lemmas.

(13) Let $E_{j}, j=1, \ldots, n$, be $n$ Banach spaces, on which the compact groups $K_{j}, j=1, \ldots, n$, act strongly continuous and isometrically. Then the direct product $K=K_{1} \times K_{2} \times \cdots \times K_{n}$ acts strongly continuous and isometrically on the projective tensor product $E=E_{1} \hat{\otimes} E_{2} \hat{\otimes} \cdots \hat{\otimes}$ $E_{n}$ and the tensor product $\left(E_{1}\right)_{K_{1}} \otimes \cdots \otimes\left(E_{n}\right)_{K_{n}}$ of the subspaces $\left(E_{j}\right)_{K_{j}}$ of $K_{j}$-invariants in $E_{j}$ is dense in the subspace $E_{K}$ of $K$-invariants in $E$.

Proof. For $x \in E_{j}, \zeta \in K_{j}$ let $x^{\zeta}$ be the image of $x$ with respect to $\zeta$. Then $x^{\#}=\int_{K_{j}} x^{\zeta} d \zeta$ is invariant and $P_{j}: x \rightarrow x^{\#}$ projects $E_{j}$ onto $\left(E_{j}\right)_{K_{j}}$. For $x=x_{1} \otimes \cdots \otimes x_{n} \in E, \zeta=\left(\zeta_{1}, \ldots, \zeta_{n}\right) \in K=K_{1} \times \cdots \times K_{n}$ we have $x^{\zeta}=x_{1}^{\zeta_{1}} \otimes \cdots \otimes x_{n}^{\zeta_{n}}$. Thus $P=P_{1} \otimes \cdots \otimes P_{n}$ projects $E$ onto $E_{K}$. Now let $z=\lim _{\mu} z_{\mu} \in E_{K}, z_{\mu} \in E_{1} \otimes \cdots \otimes E_{n}$, the algebraic tensor product. Then clearly

$$
\begin{aligned}
P z_{\mu} & \in P\left(E_{1} \otimes \cdots \otimes E_{n}\right)=\left(P_{1} E_{1}\right) \otimes \cdots \otimes\left(P_{n} E_{n}\right) \\
& =\left(E_{1}\right)_{K_{1}} \otimes \cdots \otimes\left(E_{n}\right)_{K_{n}}
\end{aligned}
$$

hence $z=P z=\lim P z_{\mu} \in\left\{\left(E_{1}\right)_{K_{1}} \otimes \cdots \otimes\left(E_{n}\right)_{K_{n}}\right\}^{-}$.

COROllaRY. Let $\mathbf{T}^{n}$ act on $\left(\mathbf{H}^{1}\right)^{\times n}$ in the obvious way, i.e. componentwise. Then $L_{\mathbf{T}^{n}}^{1}\left(\left(\mathbf{H}^{1}\right)^{\times n}\right)$ is a commutative subalgebra of $L^{1}\left(\left(\mathbf{H}^{1}\right)^{\times n}\right)$.

REMARK. It is not hard to see that $L_{\mathrm{T}^{n}}^{1}$ is even maximal commutative.

(14) Let the compact group $K$ act continuously on $G$ and let $N$ be a closed $K$-invariant subgroup of $G$; thus $K$ acts also on $G / N$. Then the canonic surjection $T: L^{1}(G) \rightarrow L^{1}(G / N)$ maps $L_{K}^{1}(G)$ onto $L_{K}^{1}(G / N)$.

This is clear, because $T$ commutes with the action of $K$, hence in particular with the projections $f \rightarrow f^{\#}, f^{\#}=\int_{K} f^{\zeta} d \zeta$.

Next we observe that the action of $K$ on $G$ defines also an action on the algebra $\mathscr{M}(G)$ of all bounded Borel measures on $G$.

(15) The subalgebra $\mathscr{M}_{K}(G)$ of all $K$-invariant measures in $\mathscr{M}(G)$ is commutative if and only if $L_{K}^{1}(G)$ is commutative.

Proof. As $L_{K}^{1}(G) \subset \mathscr{M}_{K}(G)$, we have only to show that the commutativity of $L_{K}^{1}$ implies the commutativity of $\mathscr{M}_{K}(G)$. Let $f$ and $g$ be in $L_{K}^{1}$ and $\mu$ in $\mathscr{M}_{K}$. As $L_{K}^{1}$ is an ideal in $\mathscr{M}_{K}$ we have

$$
g * \mu * f=f * g * \mu=g * f * \mu,
$$


hence $g *(\mu * f-f * \mu)=0$ for all $g \in L_{K}^{1}$ and consequently $\mu * f=f * \mu$. Now let also $\nu \in \mathscr{M}_{K}$. Then $\mu * \nu * f=\nu * f * \mu=\nu * \mu * f$ for all $f \in L_{K}^{1}$, thus $\mu * \nu=\nu * \mu$.

Corollary. For $x \in G$ let $x^{K}=\left\{x^{\xi} ; \zeta \in K\right\}$ be the K-orbit in $G$. If $L_{K}^{1}(G)$ is commutative, then $x^{K} y^{K}=y^{K} x^{K}$ for all $x, y \in G$.

Proof. For $x \in G$ define $\mu_{x} \in \mathscr{M}(G)$ by

$$
\mu_{x}(f)=\int f\left(x^{\zeta}\right) d \zeta
$$

for $f \in \mathscr{C}_{0}(G)$. Then $\mu_{x}$ is a $K$-invariant probability measure on $G$ with support $x^{K}$. Hence $\operatorname{supp}\left(\mu_{x} * \mu_{y}\right)=x^{K} y^{K}$. Now the commutativity of $L_{K}^{1}(G)$ implies $\mu_{x} * \mu_{y}=\mu_{y} * \mu_{x}$, thus $x^{K} y^{K}=y^{K} x^{K}$.

The following example will be used in the proof of Theorem 3: Let $\mathbf{H}_{\mathbf{C}}^{1}=\mathbf{C}^{3}$ be the complex 3-dimensional Heisenberg group with multiplication

$$
\left(x_{1}, x_{2}, x_{3}\right)\left(y_{1}, y_{2}, y_{3}\right)=\left(x_{1}+y_{1}, x_{2}+y_{2}, x_{3}+y_{3}+x_{2} y_{1}\right)
$$

and define an action of $\mathbf{T}^{2}$ on $\mathbf{H}_{\mathbf{C}}^{2}$ by

$$
\left(x_{1}, x_{2}, x_{3}\right)^{\left(\zeta_{1}, \zeta_{2}\right)}=\left(\zeta_{1} x_{1}, \zeta_{2} x_{2}, \zeta_{1} \zeta_{2} x_{3}\right)
$$

(16) $L_{\mathbf{T}^{2}}^{1}\left(\mathbf{H}_{\mathbf{C}}^{1}\right)$ is not commutative.

Proof. Let $x=(1,0,0), y=(0,1,0)$. Hence $x^{K}=(\mathbf{T}, 0,0), y^{K}=$ $(0, \mathbf{T}, 0), x^{K} y^{K}=(\mathbf{T}, \mathbf{T}, 0)$, but $y^{K} x^{K} \ni(0,1,0)(1,0,0)=(1,1,1) \notin x^{K} y^{K}$, hence $y^{K} x^{K} \neq x^{K} y^{K}$ and (16) follows from the last corollary.

Now we turn to the proof of Theorem 3. We consider the Lie algebra $\mathfrak{g}$ of $G$ as a $K$-module and choose some positive definite $K$-invariant bilinear form on $\mathfrak{g}$. As $z=g^{\prime}$ is $K$-invariant we can decompose $g$ into an orthogonal sum: $\mathfrak{g}=\mathrm{e} \oplus \mathrm{z}$, with $K$-invariant complement $e$ of $z$. Clearly also the complexification $g_{C}$ is a $K$-module and

$$
\mathfrak{g}_{\mathbf{C}}=\mathrm{e}_{\mathbf{C}} \oplus \mathrm{z}_{\mathbf{C}}
$$

with $z_{\mathbf{C}}=\left[e_{\mathbf{C}}, e_{\mathbf{C}}\right]$. Let $\operatorname{dim}_{\mathbf{R}} e=\operatorname{dim}_{\mathbf{C}} e_{\mathbf{C}}=m$ and let

$$
\mathrm{e}_{\mathbf{C}}=\left(z_{1}\right) \oplus\left(z_{2}\right) \oplus \cdots \oplus\left(z_{m}\right)
$$

be a decomposition of $e_{C}$ into invariant one-dimensional subspaces, hence $z_{j}^{\zeta}=\chi_{j}(\zeta) z_{j}$ for some $\chi_{j} \in \hat{K}$, the character group of $K=\mathbf{T}^{n}$. As e is $K$-invariant it follows that with $z_{j}=x_{j}+i y_{j}$ also $\bar{z}_{j}=x_{J}-i y_{j}$ is $K$-invariant, with character $\bar{\chi}_{j}$, hence $\bar{z}_{j}=z_{j}$ for some $\bar{j}$. Moreover, if $x_{j}$ and $y_{j}$ 
are linearly independent, then $\left(x_{j}\right)+\left(y_{j}\right)=e_{j}$ is an invariant irreducible subspace of $\mathrm{e}$. If on the other hand $y_{j}=0$, then necessarily $\chi_{j}=\varepsilon$, the trivial character, and $z_{j}=x_{j} \in \mathrm{e}$ is $K$-invariant. Finally, $\left[z_{j}, z_{k}\right]=w_{j, k} \in$ ${ }_{z_{C}}$ is 0 or eigenvector for the character $\chi_{j} \chi_{k}$.

(19) Assume that $w_{j, k} \neq 0$ and $\chi_{j} \chi_{k} \neq \varepsilon$ for some $j, k$. Then $L_{K}^{1}(G)$ is not commutative.

Proof. Let us assume that $z_{1}=x_{1}+i y_{1}, z_{2}=x_{2}+i y_{2}$ and $\chi_{1}, \chi_{2}$ are not trivial. Then

$$
\begin{aligned}
w & =\left[z_{1}, z_{2}\right]=\left(\left[x_{1}, x_{2}\right]-\left[y_{1}, y_{2}\right]\right)+i\left(\left[x_{1}, y_{2}\right]+\left[y_{1}, x_{2}\right]\right) \\
& =u+i v \neq 0
\end{aligned}
$$

and because $\chi_{1} \chi_{2} \neq \varepsilon$ we see that $u$ and $v$ are independent elements in $z$. Thus

$$
\mathfrak{h}=\left(x_{1}\right) \oplus\left(y_{1}\right) \oplus\left(x_{2}\right) \oplus\left(y_{2}\right) \oplus(u) \oplus(v)
$$

is a $K$-invariant subalgebra of $\mathfrak{g}$, isomorphic to the algebra of $\mathbf{H}_{\mathbf{C}}^{1}$. The corresponding subgroup $H$ in $G$ is $K$-invariant and isomorphic with $\mathbf{H}_{\mathbf{C}}^{1}$, moreover, the action of $K$ on $H$ corresponds to the action of a subgroup $T \subset \mathbf{T}^{2}$ of $\mathbf{T}^{2}$ on $\mathbf{H}_{\mathbf{C}}^{1}$. As $L_{\mathbf{T}^{2}}^{1}\left(\mathbf{H}_{\mathbf{C}}^{1}\right)$ is not commutative, see (16), $L_{T}^{1}\left(\mathbf{H}_{\mathbf{C}}^{1}\right)$ and $L_{K}^{1}(H)$ are not commutative. But $L_{K}^{1}(H) \subset \mathscr{M}_{K}(G)$, thus $L_{K}^{1}(G)$ is not commutative, see (15).

If $\chi_{1}=\varepsilon$, one can assume $z_{1}=x \in \mathrm{g}$. In this case $\chi_{2} \neq \varepsilon, z_{2}=x_{2}+$ $i y_{2} \notin \mathfrak{g}$, and

$$
\mathfrak{f}=(x) \oplus\left(x_{2}\right) \oplus\left(y_{2}\right) \oplus(u) \oplus(v)
$$

is invariant. The corresponding subgroup $F \subset G$ is isomorphic to the subgroup

$$
J=\left\{\left(z_{1}, z_{2}, z_{3}\right) \in \mathbf{H}_{\mathbf{C}}^{1} ; z_{1} \in \mathbf{R}\right\}
$$

with action $\left(z_{1}, z_{2}, z_{3}\right)^{\zeta}=\left(z_{1}, \zeta z_{2}, \zeta z_{3}\right)$ for $\zeta \in \mathbf{T}$. Analogously to (16) one proves that $L_{\mathrm{T}}^{1}(J)$, hence $L_{K}^{1}(F)$ and again $L_{K}^{1}(G)$ are not commutative.

Now we will study the case in which $\mathbf{T}^{n}$ acts trivially on the center $\mathfrak{z}=\mathfrak{g}^{\prime}$ of $\mathfrak{g}$ and $2 n<\operatorname{dim}(\mathfrak{g} / \mathfrak{z})$. We will show that in this case $L_{\mathrm{T}^{n}}^{1}(G)$ is not commutative by reducing the problem to the special case in which $\mathbf{T}^{n}$ acts on $\mathfrak{h}^{m}, m>n$, as a subgroup of the "standard action" of $\mathbf{T}^{m}$ on $\mathfrak{h}^{m}$, resp. $\mathbf{H}^{m}$ as defined in (7) or (8), or $n=1, m=2$.

(20) If $z=\mathfrak{g}^{\prime}$ and $\operatorname{dim}(\mathfrak{g} / \mathfrak{z})=d \geqq 4$, then $g$ has a quotient $\mathfrak{g} / \mathfrak{f}$ isomorphic to $\mathfrak{h}^{j}$ for $j \geq 2$. 
Proof. Let $z_{0}$ be a subspace of $z$ of codimension 1, thus $z=(z) \oplus z_{0}$ with some $z \neq 0$ in $z$. As $z=g^{\prime}$ we can assume that $z=[x, y]$ for $x$, $y \in \mathfrak{g}$. This implies that $\overline{\mathrm{g}}=\mathrm{g} / \mathrm{z}_{0}$ has one dimensional center, consequently $\overline{\mathfrak{g}} \cong \mathfrak{h}^{l} \oplus$ a with $l \geq 1$ and central ideal $\mathfrak{a}$. It follows in particular that $(20)$ is true if $\operatorname{dim} z=1$. Now let us assume that $\operatorname{dim} z>1$. In this case we can find $x_{1}, y_{2}, x, y$ in $g$ with $\left[x_{1}, y_{1}\right]=z_{1},[x, y]=z, z_{1}$ and $z$ independent in $z$.

If $x_{1}, y_{1}, x$ and $y$ are linearly independent modulo $z$ we set $x=x_{2}$, $y=y_{2}, z=z_{2}$ and $z=\left(z_{1}\right) \oplus\left(z_{2}\right) \oplus z_{0}$. If $x_{1}, y_{1}, x$ and $y$ are not independent modulo $z$, then $\operatorname{dim}\left(x_{1}, y_{1}, x, y\right) / z=3$ and it is easy to see that in this case one can assume $x=x_{1}$. By hypothesis there exists $q \in \mathfrak{g}$, independent $(\bmod z)$ of $x_{1}, y_{1}, y$.

If $[q, y] \notin\left(z_{1}\right)$ we set $x_{2}=q, y_{2}=y$ and $z_{2}=\left[x_{2}, y_{2}\right]$.

If $[q, y]=\alpha z_{1}, \alpha \in \mathbf{R}$, we set $x_{2}=x_{1}+q, y_{2}=y, z_{2}=\left[x_{2}, y_{2}\right]=$ $\left[x_{1}, y\right]+[q, y]=z+\alpha z_{1} \notin\left(z_{1}\right)$.

Again $z=\left(z_{1}\right) \oplus\left(z_{2}\right) \oplus z_{0}$, thus in all cases the quotient $g / z_{0}$ contains a 6-dimensional ideal $\mathfrak{f}$, generated by the cosets $x_{j}, y_{k} \bmod \mathfrak{z}$, which we denote also by $x_{j}, y_{k}$, with $\left[x_{1}, y_{1}\right]=z_{1},\left[x_{2}, y_{2}\right]=z_{2}, z(=$ center of $\mathfrak{f})$ $=\left(z_{1}\right) \oplus\left(z_{2}\right)$.

As above we see that $\mathfrak{f} /\left(z_{j}\right) \cong \mathfrak{h}^{l_{j}} \oplus \mathfrak{a}_{j}$ with central $\mathfrak{a}_{j}$. If $l_{1}=2$ or $l_{2}=2, \mathfrak{f}$, and consequently $\mathfrak{g}$, has a quotient isomorphic to $\mathfrak{h}^{l}$ with $l \geq 2$. Otherwise the projections

$$
p_{j}: \mathfrak{f} /\left(z_{j}\right) \cong \mathfrak{h}^{1} \oplus \mathfrak{a}_{j} \rightarrow \mathfrak{h}^{1}
$$

define homomorphisms $P_{j}: \mathfrak{f} \rightarrow \mathfrak{h}^{1}$ with $\left(\operatorname{ker} P_{j}\right) \cap \mathfrak{z}=\left(z_{j}\right)$, hence ker $P_{1}$ $\cap \operatorname{ker} P_{2}=0$. It follows that

$$
P=P_{1} \oplus P_{2}: \mathfrak{f} \rightarrow \mathfrak{h}^{1} \oplus \mathfrak{h}^{1}
$$

is injective. As $\operatorname{dim} \mathfrak{f}=\operatorname{dim}\left(\mathfrak{h}^{1} \oplus \mathfrak{h}^{1}\right)$ it also follows that $P$ is an isomorphism. Let $\left(c_{1}\right) \oplus\left(c_{2}\right)$ be the center of $\mathfrak{h}^{1} \oplus \mathfrak{h}^{1}$. Then $\left(\mathfrak{h}^{1} \oplus \mathfrak{h}^{1}\right) /\left(c_{1}-c_{2}\right)$ $\cong \mathfrak{h}^{2}$, hence also $\mathfrak{f}$ has a quotient isomorphic to $\mathfrak{h}^{2}$. This proves $(20)$.

(21) Let $\mathbf{T}$ act faithfully on $\mathfrak{h}^{2}$. There exists a basis $\left\{x_{1}, y_{1}, x_{2}, y_{2}, z\right\}$ of $\mathfrak{h}^{2}$ with $\left[x_{j}, y_{k}\right]=\delta_{j k} z$, such that the action of $\mathbf{T}$ on $\mathfrak{h}^{2}$ is defined either by by

(i) $z_{j}^{\zeta}=\zeta^{r_{j}} z_{j}, z^{\zeta}=z$ with $z_{j}=x_{j}+i y_{j} \in \mathfrak{h}_{\mathbf{C}}^{2}$, and $r_{j} \in \mathbf{Z}, r_{1} \neq 0$, or

(ii) $\left(x_{1}+i x_{2}\right)^{\zeta}=\zeta^{r}\left(x_{1}+i x_{2}\right),\left(y_{1}+i y_{2}\right)^{\zeta}=\zeta^{r}\left(y_{1}+i y_{2}\right)$ with nonzero $r \in \mathbf{Z}$.

Proof. As T acts nontrivially on $\mathfrak{h}^{2}$, hence also on the complexification $\mathfrak{h}_{\mathbf{C}}^{2}$, the latter contains an $a=u+i v$ with $a^{\zeta}=\zeta^{r} a$ for some $r \in \mathbf{Z}$, $r \neq 0$. Then $\bar{a}=u-i v$ satisfies $\bar{a}^{\zeta}=\zeta^{-r} \bar{a}$. Let $e_{1}=(u) \oplus(v) \subset \mathfrak{h}^{2}$. 
(i) Assume that there exists a $\mathbf{T}$-invariant subspace $e_{2}$ in $\mathfrak{h}^{2}$ with $\mathfrak{h}^{2}=e_{1} \oplus e_{2} \oplus(z)$ and $\left[e_{1}, e_{2}\right]=0$. Then obviously $\left[e_{1}, e_{1}\right]=\left[e_{2}, e_{2}\right]=$ $(z)$, hence $e_{2} \oplus(z) \cong \mathfrak{h}^{1}$ and we can find $x_{2}, y_{2}$ in $e_{2}$ with $\left[x_{2}, y_{2}\right]=z$, $\left(x_{2}+i y_{2}\right)^{\zeta}=\zeta^{r_{2}}\left(x_{2}+i y_{2}\right), r_{2} \in \mathbf{Z}$. Now also $\left[\mathrm{e}_{1}, \mathrm{e}_{1}\right]=(z)$, thus we can normalize $u$ and $v$ so that $[u, v]=\varepsilon z$ with $\varepsilon= \pm 1$. Putting $x_{1}=u$, $y_{1}=\varepsilon v, r_{1}=\varepsilon r$ we see that we have case (i).

(ii) In the other case there exists $b \in \mathfrak{h}_{\mathbf{C}}^{2}$, linearly independent of $a$ and $\bar{a}$, with $[a, b]=z, b^{\zeta}=\zeta^{r^{\prime}} b$. As $z^{\zeta}=z$ it follows that $r^{\prime}=-r$. Hence $b \neq \bar{b},[a, \bar{b}]=[\bar{a}, b]=0$ and $\{a, \bar{a}, b, \bar{b}, z\}$ is a basis for $\mathfrak{h}_{\mathbf{C}}^{2}$.

Let $[a, \bar{a}]=\alpha z$. Then $c=\bar{a}-\alpha b$ also satisfies $c^{\zeta}=\zeta^{-r} c,[a, c]=0$, $[\bar{a}, c]=0$. But this implies $\left[e_{1}, e_{2}\right]=0$ for $e_{2}=((c)+(\bar{c})) \cap \mathfrak{h}^{2}$, which is excluded in case (ii), unless $\alpha=0$. Thus $[a, \bar{a}]=0$ and similarly $[b, \bar{b}]=0$. Putting $2 a=\left(x_{1}+i x_{2}\right), \bar{b}=\left(y_{1}+i y_{2}\right)$, we see that (20)(ii) holds.

Now we return to the general case and consider again the decompositions (17) and (18). Moreover we assume that $K=\mathbf{T}^{n}$ acts trivially on $z$. If $\mathfrak{f}_{\chi}=\Sigma_{\chi_{j}=\chi}\left(z_{j}\right) \subset e_{\mathbf{C}}$, we have

$$
\mathrm{e}_{\mathbf{C}}=\sum_{\chi}^{\oplus} \mathfrak{f}_{\chi}, \quad\left[\mathfrak{f}_{\chi}, \mathfrak{f}_{\Psi}\right]=0 \quad \text { for } \chi \neq \bar{\Psi}
$$

and $\mathfrak{f}_{\chi} \neq 0$ implies $\mathfrak{z}_{\chi}=\left[\mathfrak{f}_{\chi}, \mathfrak{f}_{\bar{\chi}}\right] \cap \mathfrak{g} \neq 0$

(22) If $\operatorname{dim}_{\mathbf{C}} \mathfrak{f}_{\chi} \geq 2$ for some $\chi \in \hat{K}$, then $L_{K}^{1}(G)$ is not commutative.

Proof. Assume e.g. that $\mathfrak{f}_{\chi}=\left(z_{1}\right) \oplus\left(z_{2}\right)$ with $z_{j}=x_{j}+i y_{j}, z_{j}^{\zeta}=$ $\chi(\zeta) z_{j}, j=1,2$. Then

$$
\mathfrak{g}_{\chi}=\left(\mathfrak{f}_{\chi} \oplus \mathfrak{f}_{\bar{\chi}} \oplus \mathfrak{z}_{\chi}\right) \cap \mathfrak{g}
$$

is an invariant ideal in $\mathfrak{g}$ with $\mathfrak{g}_{\chi}^{\prime}=z_{\chi}$. Let $G_{\chi}$ be the corresponding invariant normal subgroup in $G$. If $\chi=\varepsilon$, then $K$ acts trivially on $G_{\chi}$, hence $L_{K}^{1}\left(G_{\chi}\right)=L^{1}\left(G_{\varepsilon}\right)$ is not commutative. If $\chi \neq \varepsilon$, then $K / \operatorname{ker} \chi \cong \mathbf{T}$ acts on $G_{\chi}$. It follows from (20) that $G_{\chi}$ has a $\mathbf{T}$-invariant normal subgroup $N$ with $G_{\chi} / N=F \cong \mathbf{H}^{2}$ and $\mathbf{T}$ acts on $F$ as described in (21). We will show that this implies the noncommutativity of $L_{K}^{1}(F)=L_{\mathrm{T}}^{1}(F)$, hence also of $L_{K}^{1}\left(G_{\chi}\right)$ and $L_{K}^{1}(G)$. To realize (21)(ii) on the group level let $\mathbf{H}^{2}=\mathbf{C}^{2} \times \mathbf{R}$ with multiplication

$$
\left(u_{1}, u_{2}, r\right)\left(v_{1}, v_{2}, s\right)=\left(u_{1}+v_{1}, u_{2}+v_{2}, r+s+\frac{1}{2} \operatorname{Re}\left(u_{1} \bar{v}_{2}-u_{2} \bar{v}_{1}\right)\right)
$$

and action $\left(u_{1}, u_{2}, r\right)^{\zeta}=\left(\zeta u_{1}, \zeta u_{2}, r\right)$. Let $x_{1}=(1,0,0)$ and $x_{2}=(0,1,0)$. Then

$$
\begin{aligned}
& x_{1}^{\mathbf{T}} x_{2}^{\mathbf{T}}=\left\{\left(\zeta_{1}, \zeta_{2}, \frac{1}{2} \operatorname{Re} \zeta_{1} \bar{\zeta}_{2}\right)\right\} \ni\left(1,1, \frac{1}{2}\right) \\
& x_{2}^{\mathbf{T}} x_{1}^{\mathbf{T}}=\left\{\left(\zeta_{2}, \zeta_{1},-\frac{1}{2} \operatorname{Re} \zeta_{2} \bar{\zeta}_{1}\right)\right\} \nexists\left(1,1, \frac{1}{2}\right)
\end{aligned}
$$


i.e. the orbits of $x_{1}$ and $x_{2}$ don't commute. As before this implies that $L_{\mathbf{T}}^{1}\left(\mathbf{H}^{2}\right)$, hence $L_{K}^{1}(G)$ is not commutative.

The case (21)(i) is covered by the more general following lemma (23). Let $\mathbf{T}^{n}$ act on $\mathbf{H}^{n}$ as defined by (6) or (7). If $K$ is a closed proper subgroup of $\mathbf{T}^{n}$, then $L_{K}^{1}\left(\mathbf{H}^{n}\right)$ is not commutative.

Proof. We identify the character group $\hat{\mathbf{T}}^{n}$ with $\mathbf{Z}^{n}$ and write $\hat{q}=$ $\left(q_{1}, \ldots, q_{n}\right) \in \mathbf{Z}^{n}$ for the character $\hat{q}(\zeta)=\prod_{1}^{n} \zeta_{j}^{q_{j}}$. As $K \neq \mathbf{T}^{n}$ there exists some $\hat{q} \neq \varepsilon=(0, \ldots, 0)$ with $\hat{q}(K)=\{1\}$. Let $\hat{q}=\hat{a}-\hat{b}$ with $\hat{a}, \hat{b} \in \mathbf{N}^{n}$. Then $\left.\left.\hat{a}\right|_{K} \equiv \hat{b}\right|_{K}=\Psi \in \hat{K}$.

After Theorem 2 we have $A\left(\mathbf{R}^{n}\right)_{1}=\left(\sum_{\mathbf{N}^{n}}^{\oplus}\left(u_{\hat{q}}\right)\right)^{-}$with respect to the action of $\mathbf{T}^{n}$ on $A\left(\mathbf{R}^{n}\right)_{1}$. If $A_{1}^{\chi}$ is the $\chi$-primary component of $A\left(\mathbf{R}^{n}\right)_{1}$ with respect to the action of $K$ it follows that $A_{1}^{\Psi}$ contains $u_{\hat{a}}$ and $u_{\hat{b}}$, thus $\operatorname{dim} A_{1}^{\Psi}>1$. Now (4) implies that $\Gamma\left(\mathbf{R}^{n}\right)^{\#}$ and $L_{K}^{1}\left(\mathbf{H}^{n}\right)$ are not commutative. This also proves (23).

Now assume that $L_{K}^{1}(G)$ is commutative, $K=\mathbf{T}^{n}$. Then (22) implies that $\operatorname{dim} \mathfrak{f}_{\chi} \leq 1$ for all $\chi \in \hat{K}$, thus $\mathfrak{f}_{\chi}=\left(z_{\chi}\right)$ with $z_{\chi}=x_{\chi}+i y_{\chi}, \mathrm{e}_{\chi}=$ $\left(x_{\chi}\right) \oplus\left(y_{\chi}\right), w_{\chi}=\left[x_{\chi}, y_{\chi}\right] \in z,\left[e_{\chi}, e_{\Psi}\right]=0$ for $\chi \neq \Psi$. This implies $\mathfrak{f}_{\varepsilon}=$ 0 , because $\mathfrak{f}_{\varepsilon}=\mathfrak{f}_{\bar{\varepsilon}}$, hence $e_{\varepsilon}=\left(x_{\varepsilon}\right) \subset z$, thus $e_{\varepsilon}=0$. The same argument shows that $w_{\chi} \neq 0$ if $e_{\chi} \neq 0$. Now it is clear that $G$ has a quotient isomorphic to $\mathbf{H}^{m}$, where $m=\frac{1}{2} \operatorname{dim}(g / z)$. Let $\chi_{1}, \ldots, \chi_{m}$ be the characters of $K$ for which $e_{j}=e_{\chi} \neq 0$. Then $\iota: \zeta \rightarrow\left\{\chi_{1}(\zeta), \ldots, \chi_{m}(\zeta)\right\} \in \mathbf{T}^{m}$ is an injection from $K$ into $\mathbf{T}^{m}$ and if $x \rightarrow x^{\xi}$ is the given action of $K$ on $G$, resp. the quotient $\mathbf{H}^{m}$ of $G$, and $x \rightarrow x^{\bullet \xi}, \xi \in \mathbf{T}^{m}$, is the standard action, we clearly have $x^{\zeta}=x^{\cdot \bullet(\zeta)}$. It follows now from $(23)$ that $\iota(K)=$ $\mathbf{T}^{m}$, hence $n=m$. Thus we have shown the necessity of the conditions in Theorem 3.

Now let $K=\mathbf{T}^{n}$ and $\operatorname{dim}(g / z)=2 n$. We consider again the decomposition of $e_{\mathbf{C}}$ into primary components $\mathfrak{f}_{\chi}, \chi \in \hat{K}=\mathbf{Z}^{n}$. As $\mathfrak{f}_{\chi} \neq 0$ always implies $\mathfrak{f}_{\bar{\chi}} \neq 0$ we can write

$$
\mathrm{e}_{\mathbf{C}}=\mathfrak{f}_{\varepsilon} \oplus \sum_{j=1}^{r} \oplus \mathfrak{f}_{\chi_{j}} \oplus \sum_{j=1}^{r} \oplus \mathfrak{f}_{\bar{\chi}}, \quad \mathfrak{f}_{\chi_{j}} \neq 0 .
$$

As $\cap_{j=1}^{r} \operatorname{ker} \chi_{j}=\{1\}$ it follows that the $\chi_{j}$ generate $\mathbf{Z}^{n}$, hence $r=n$, $\mathfrak{f}_{\varepsilon}=0, \mathfrak{f}_{\chi_{j}}=\left(z_{j}\right)$. Moreover, $\zeta \rightarrow\left\{\chi_{1}(\zeta), \chi_{2}(\zeta), \ldots, \chi_{n}(\zeta)\right\}$ is an automorphism of $\mathbf{T}^{n}$ and consequently we can assume that $\chi_{j}(\zeta)=\zeta_{j}$ for $\zeta=\left\{\zeta_{1}, \zeta_{2}, \ldots, \zeta_{n}\right\} \in \mathbf{T}^{n}$. This means that $z_{j}^{\zeta}=\zeta_{j} z_{j}$. If $z_{j}=x_{j}+i y_{j}$, then $\left[x_{j}, y_{j}\right]=w_{j} \neq 0,\left(x_{j}\right) \oplus\left(y_{j}\right) \oplus\left(w_{j}\right)=\mathfrak{h}_{j}$ is an ideal, isomorphic with $\mathfrak{h}^{1}$ 
and $\mathfrak{g}$ is canonically an image of the direct sum $\left(\mathfrak{h}^{1}\right)^{\oplus n}=\mathfrak{h}_{1} \oplus \mathfrak{h}_{2} \oplus$ $\cdots \oplus \mathfrak{h}_{n}$. Correspondingly $G$ is a quotient of $\left(\mathbf{H}^{1}\right)^{\times n}=\mathbf{H}^{1} \times \mathbf{H}^{1} \times$ $\cdots \times \mathbf{H}^{1}$. Moreover, the epimorphism $\left(\mathbf{H}^{1}\right)^{\times n} \rightarrow G$ commutes with the "canonic" action of $\mathbf{T}^{n}$ on $\left(\mathbf{H}^{1}\right)^{\times n}$ and the given action of $\mathbf{T}^{n}$ on $G$. Because $L_{\mathbf{T}^{n}}^{1}\left(\left(\mathbf{H}^{1}\right)^{\times n}\right)$ is commutative (corollary of (13)), (14) yields that also $L_{\mathbf{T}^{n}}^{1}(G)$ is commutative. Thus Theorem 3 is completely proved.

It is now easy to prove that $L_{K}^{1}(G)$ for connected compact abelian $K$ and connected nilpotent $G$ is never commutative if $G$ is of class greater than 2:

THEOREM 4. If $G$ is a connected nilpotent Lie group and $K$ connected compact abelian, such that $L_{K}^{1}(G)$ is commutative, then $G$ is at most of class 2, hence of the form described in Theorem 3.

Proof. A similar argument as the one before Theorem 3 shows that we may assume $z \subset \mathfrak{g}^{\prime}$. Then instead of (17) we have the $K$-invariant decomposition $\mathfrak{g}_{\mathbf{C}}=\mathrm{e}_{\mathbf{C}} \oplus \mathrm{g}_{\mathbf{C}}^{\prime}$ and for $\mathrm{e}_{\mathbf{C}}$ again the decomposition (18). Moreover, also (19) holds: Let $p$ be minimal such that $w_{j, k}=w=u+i v$ is not contained in the $p$ th term $g_{C}^{(p)}$ of the lower central series. Then with the notation of (19), $\mathfrak{h}$ is an invariant subalgebra modulo $\mathfrak{g}^{(p)}$, hence the quotient $G_{p}$ of $G$ modulo $\exp g^{(p)}$ contains an $K$-invariant subgroup isomorphic with $H_{\mathrm{C}}^{1}$ or with $J$ in the second case of (19). But then (15) implies that $L_{K}^{1}\left(G_{p}\right)$ is not commutative and from (14) the same follows for $L_{K}^{1}(G)$. Thus if $L_{K}^{1}(G)$ is commutative we have $\left[z_{j}, z_{k}\right]=0$ for all $z_{j}$, $z_{k} \in \mathrm{e}_{\mathbf{C}}$ which transform under $K$ with characters $\chi_{j}, \chi_{k}$ with $\chi_{k} \neq \bar{\chi}_{j}$. But this implies that $K$ acts trivially on all $[x, y]$ with $x, y \in \mathrm{e}_{\mathbf{C}}$. As $\mathrm{e}_{\mathbf{C}}$ generates $\mathrm{g}_{\mathbf{C}}$ it follows that $K$ acts trivially on $\mathrm{g}_{\mathbf{C}}^{\prime}$.

From $z \subset g^{\prime}$ we conclude as above that $e_{C}$ cannot contain nonzero $K$-fixed elements, in particular the $\chi_{j} \in \hat{K}$, corresponding to $z_{j}$ in (18) is not trivial. This implies $\left[z_{j}, w\right]=0$ for all $w \in g_{\mathbf{C}}^{\prime}$, because $K$ is trivial on $\mathfrak{g}_{\mathbf{C}}^{\prime}$. We conclude that $\left[\mathrm{e}_{\mathbf{C}}, \mathfrak{g}_{\mathbf{C}}^{\prime}\right]=0$, thus $\left[\mathrm{e}, \mathfrak{g}^{\prime}\right]=0$ and as $\mathrm{e}$ generates $\mathfrak{g}$, finally also $\left[\mathfrak{g}, \mathfrak{g}^{\prime}\right]=0$, which finishes the proof of Theorem 4 .

\section{REFERENCES}

[1] D. Geller, Fourier analysis on the Heisenberg group. I, Schwartz space, J. Funct. Anal., 36 (1980), 205-254.

[2] R. Howe, Quantum mechanics and partial differential equations, J. Funct. Anal., 38 (1980), 188-254.

[3] On the role of the Heisenberg group in harmonic analysis, Bull. Amer. Math. Soc., (N.S.) 3 (1980), 821-843.

[4] A. Hulanicki and F. Ricci, A Tauberian theorem and tangential convergence for bounded harmonic functions on balls in $\mathbf{C}^{n}$, Inventiones Math., 62 (1980), 325-331. 
[5] A. Korányi, Some applications of Gelfand pairs in classical analysis, Harmonic analysis and group representations, C.I.M.E., 1980, Liguori Editore, Napoli (1982), 333-348.

[6] H. Leptin, Darstellungen verallgemeinerter L ${ }^{1}$-Algebren, Inventiones Math., 5 (1968), $192-215$.

[7] _ - On group algebras of nilpotent groups, Studia Math., 47 (1973), 37-49.

[8] Ideal theory in group algebras of locally compact groups, Inventiones Math., 31 (1976), 259-278.

[9] On onesided harmonic analysis in noncommutative locally compact groups, J. Reine u. Angewandte Math., 306 (1979), 122-153.

[10] _ Bemerkungen über Linksideale in Gruppenalgebren, Lecture Notes in Mathematics, 781 (1980), 121-141.

[11] H. Leptin and D. Poguntke, Symmetry and nonsymmetry for locally compact groups, J. Funct. Anal., 33 (1979), 119-134.

[12] D. Poguntke, Gewisse Segalsche Algebren auf lokal kompakten Gruppen, Archiv Math., 33 (1979), 454-460.

[13] F. Ricci, Harmonic analysis on generalized Heisenberg groups, preprint Torino (1982).

Received February 23, 1983.

UNIVERSITÄT BIELEFELD

UNIVERSITÄTSSTRAßE

4800 BieleFELD, FeD. REP. GERMANY 



\section{PACIFIC JOURNAL OF MATHEMATICS \\ EDITORS}

Donald BABBITT (Managing Editor)

University of California

Los Angeles, CA 90024

J. Dugundu

University of Southern Californa

Los Angeles, CA 90089-1113

R. FINN

Stanford University

Stanford, CA 94305

HermanN FLaSChKa

University of Arizona

Tucson, AZ 85721

\author{
C. C. MOORE \\ University of California \\ Berkeley, CA 94720 \\ ARTHur Ogus \\ University of California \\ Berkeley, CA 94720 \\ Hugo Rossi \\ University of Utah \\ Salt Lake City, UT 84112 \\ H. SAMELSON \\ Stanford University \\ Stanford, CA 94305
}

ASSOCIATE EDITORS
R. ARENS
E. F. BECKENBACH
B. H. NeUmanN
F. WOLF
K. YOSHIDA (1906-1982)

\section{SUPPORTING INSTITUTIONS}

UNIVERSITY OF ARIZONA

UNIVERSITY OF BRITISH COLUMBIA

UNIVERSITY OF OREGON

CALIFORNIA INSTITUTE OF TECHNOLOGY

UNIVERSITY OF CALIFORNIA

MONTANA STATE UNIVERSITY

UNIVERSITY OF SOUTHERN CALIFORNIA

UNIVERSITY OF NEVADA, RENO

STANFORD UNIVERSITY

UNIVERSITY OF HAWAII

NEW MEXICO STATE UNIVERSITY

UNIVERSITY OF TOKYO

UNIVERSITY OF UTAH

WASHINGTON STATE UNIVERSITY

OREGON STATE UNIVERSITY

UNIVERSITY OF WASHINGTON 


\section{Pacific Journal of Mathematics}

\section{Vol. 116, No. $1 \quad$ November, 1985}

K. Adachi, Le problème de Lévi pour les fibrés grassmanniens et les variétés

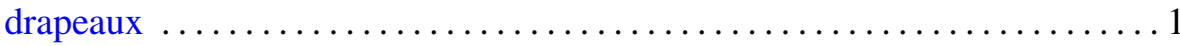

John MacLeod Ball, Remarks on the paper: "Basic calculus of variations" . . . 7 John Kelly Beem and Phillip E. Parker, Whitney stability of solvability . . . 11 Alberto Facchini, Decompositions of algebraically compact modules .......25

S. S. Khare, Finite group action and equivariant bordism $\ldots \ldots \ldots \ldots . \ldots 39$

Horst Leptin, A new kind of eigenfunction expansions on groups $\ldots \ldots \ldots . .45$

Pei-Kee Lin, Unconditional bases and fixed points of nonexpansive

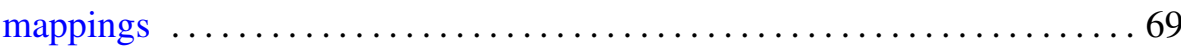

Charles Livingston, Stably irreducible surfaces in $S^{4} \ldots \ldots \ldots \ldots \ldots 77$

Kevin Mor McCrimmon, Nonassociative algebras with scalar involution . . .885

Albert Milani, Singular limits of quasilinear hyperbolic systems in a

bounded domain of $\mathbf{R}^{3}$ with applications to Maxwell's equations

Takemi Mizokami, On $M$-structures and strongly regularly stratifiable

spaces

Jesper M. Møller, On the homology of spaces of sections of complex

projective bundles

Nikolaos S. Papageorgiou, Carathéodory convex integrand operators and

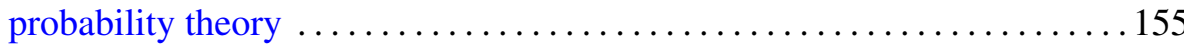

Robert John Piacenza, Transfer in generalized prestack cohomology 185

Lance W. Small and Adrian R. Wadsworth, Integrality of subrings of matrix rings ...

James Michael Wilson, On the atomic decomposition for Hardy spaces 